Linköping Studies in Science and Technology

Thesis No. 1713

\title{
MRI based radiotherapy planning and pulse sequence optimization
}

\author{
Jens Sjölund
}

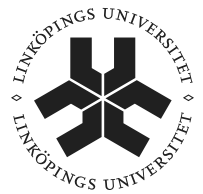

Linköping University

INSTITUTE OF TECHNOLOGY

Department of Biomedical Engineering

Linköping University, Sweden

Linköping, April 2015 
MRI based radiotherapy planning and pulse sequence optimization

\author{
(C) 2015 Jens Sjölund
}

Department of Biomedical Engineering

Linköping University, Sweden 


\section{Abstract}

Radiotherapy plays an increasingly important role in cancer treatment, and medical imaging plays an increasingly important role in radiotherapy. Magnetic resonance imaging (MRI) is poised to be a major component in the development towards more effective radiotherapy treatments with fewer side effects. This thesis attempts to contribute in realizing this potential.

Radiotherapy planning requires simulation of radiation transport. The necessary physical properties are typically derived from CT images, but in some cases only MR images are available. In such a case, a crude but common approach is to approximate all tissue properties as equivalent to those of water. In this thesis we propose two methods to improve upon this approximation. The first uses a machine learning algorithm to automatically identify bone tissue in MR. The second, which we refer to as atlas-based regression, can be used to generate a realistic, patient-specific, pseudo-CT directly from anatomical MR images. Atlas-based regression uses deformable registration to estimate a pseudo-CT of a new patient based on a database of aligned MR and CT pairs.

Cancerous tissue has a different structure from normal tissue. This affects molecular diffusion, which can be measured using MRI. The prototypical diffusion encoding sequence has recently been challenged with the introduction of more general gradient waveforms. To take full advantage of their capabilities it is, however, imperative to respect the constraints imposed by the hardware while at the same time maximizing the diffusion encoding strength. In this thesis we formulate this as a constrained optimization problem that is easily adaptable to various hardware constraints. 



\section{Acknowledgements}

In popular culture, research is often depicted as a lonely endeavor. Let me tell you that it is not-at least not in my case. There are a number of people to whom I would like to express my gratitude.

Many thanks goes to my main supervisor Hans Knutsson, for showing me the traits and gaits of a successful researcher. Thanks to my co-supervisor Mats Andersson, for allowing me to put forward this thesis despite never winning a set. You are a silent hero. The help of Anders Eklund in writing this thesis is also acknowledged.

The Swedish Research Council is acknowledged for funding part of this research as an industrial $\mathrm{PhD}$ project together with Elekta, where I am fortunate to work.

I would like to extend my gratitude to the research and physics group at Elekta, for their expertise, curiosity and friendship; to Jonas Gårding, for playing the long game in a time of quarterly reports and to Håkan Nordström, for his fondness of pastries.

My warmest appreciations go to my parents Eva and Tomas Sjölund, for always having my back, and to Barbro Gustafsson, for never losing interest.

And finally, to Maria, for bearing with me, and being a constant reminder of what really matters.

Jens Sjölund

Linköping, March 2015 



\section{Table of Contents}

1 Introduction $\quad 1$

1.1 Outline ........................ 1

1.2 Publications . . . . . . . . . . . . . . . 3

1.3 Abbreviations ................... 4

2 Radiotherapy $\mathbf{5}$

2.1 Radiobiology . . . . . . . . . . . . . . 5

2.1.1 Cellular damage caused by ionizing radiation . . . . 5

2.1.2 The five R's of radiobiology . . . . . . . . . . 7

2.2 Radiotherapy modalities . . . . . . . . . . . . . . . . 11

2.2.1 Stereotactic radiosurgery . . . . . . . . . . . 12

2.3 Physics of Cobalt-60 radiation . . . . . . . . . . . . 13

2.3.1 Energy deposition in photon beams . . . . . . . . . 14

2.4 Treatment planning . . . . . . . . . . . . . . . . . 15

2.4.1 Dose calculations . . . . . . . . . . . . . . . 15

3 Magnetic resonance imaging $\quad 17$

3.1 The origin of the MR signal . . . . . . . . . . . . . 18

3.2 Excitation and relaxation . . . . . . . . . . . . . . . 20

3.3 Pulse sequences . . . . . . . . . . . . . . . . . . . . . 23

3.3 .1 Gradients . . . . . . . . . . . . . . . 23

3.3 .2 Echoes . . . . . . . . . . . . . . . . 24

3.3.3 Pulse parameters . . . . . . . . . . . . . . 24

3.4 Signal localization . . . . . . . . . . . . . . . . . 25

4 Diffusion MRI $\quad 29$

4.1 Diffusion . . . . . . . . . . . . . . . . . . . . . . . 29

4.1.1 Statistical ensembles . . . . . . . . . . . 30

4.2 Stochastic process . . . . . . . . . . . . . . 31

4.2 .1 Autocorrelation function . . . . . . . . . . . . 31

4.2.2 Diffusivity and autocorrelation functions . . . . . . . 31

4.2 .3 The diffusion tensor . . . . . . . . . . . . . . 32

4.3 Measuring diffusion with MRI . . . . . . . . . . . . . 33

4.3.1 Eulerian and Lagrangian description of flow . . . . . 34

4.3.2 The Bloch-Torrey equation . . . . . . . . . . 34

4.4 The Stejskal-Tanner experiment . . . . . . . . . . . . . 35

4.4.1 The narrow pulse approximation . . . . . . . 37 
4.4.2 The apparent diffusion coefficient . . . . . . . . 38 


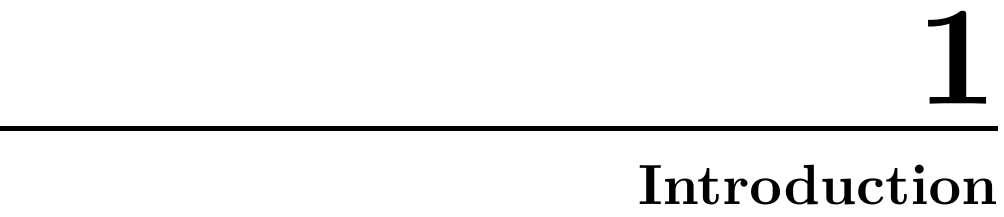

"SSS-Start Simple, Stupid!"

Thomas Schön (1977-)

Cancer. The word itself seems frightening. One in three Swedes is expected to develop cancer at some point [56]. Yet, there is hope. Taken over all cancer types, the 5-year survival ratio relative to the general population is about $65 \%$ - and improving [66,67]. Surgery is the most common treatment form, but radiotherapy plays an increasingly important role $[48,56]$.

Improvements in radiotherapy, in turn, go hand in hand with those in medical imaging. Today's radiotherapy workflow is based on X-ray computed tomography (CT). Magnetic resonance imaging (MRI) is, however, rapidly being introduced due to its superior soft tissue contrast and the possibility of using it to image physiological processes [65]. Although MRI is likely to - by and large - improve radiotherapy, it is not without its own set of uncertainties. It is my humble hope that this thesis will contribute to reducing these uncertainties.

Upon reading this thesis you will be taken on a tour-or what might at times seem like a detour - of the technical details of magnetic resonance imaging and radiotherapy. There is a risk that we forget why we are doing this. At such times, we must remind ourselves not to lose sight of our ultimate goal-to cure cancer.

\subsection{Outline}

This thesis consists of a background part followed by three research papers. The background part, in turn, consists of three chapters. Chapter 2 provides a general background on radiotherapy: from the principles of how ionizing radiation interacts with biological matter to how treatment plans are designed. Chapter 3 covers the physics behind the magnetic resonance 
phenomena and how it can be used to generate images with various contrasts. Finally, chapter 4 describes how magnetic resonance can be used to probe translational motion on the microscopic level. 


\subsection{Publications}

I J. Sjölund, A. Eriksson Järliden, M. Andersson, H. Knutsson and H. Nordström. Skull Segmentation in MRI by a Support Vector Machine Combining Local and Global Features. In 22nd International Conference on Pattern Recognition (ICPR), pages 3274-3279. IEEE, 2014.

II J. Sjölund, D. Forsberg, M. Andersson, and H. Knutsson. Generating patient specific pseudo-CT of the head from MR using atlas-based regression. Physics in medicine and biology 60(2):825, 2015.

III J. Sjölund, M. Nilsson, D. Topgaard, C-F. Westin, and H. Knutsson. Constrained optimization of gradient waveforms for generalized diffusion encoding. In preparation. 


\subsection{Abbreviations}

This table lists some of the abbreviations that are used in this thesis, along with their meanings.

$\begin{array}{ll}\text { ADC } & \text { Apparent Diffusion Coefficient } \\ \text { CPU } & \text { Central Processing Unit } \\ \text { CSF } & \text { Cerebrospinal Fluid } \\ \text { CT } & \text { Computed Tomography } \\ \text { dMRI } & \text { Diffusion Magnetic Resonance Imaging } \\ \text { DTI } & \text { Diffusion Tensor Imaging } \\ \text { FFT } & \text { Fast Fourier Transform } \\ \text { FID } & \text { Free Induction Decay } \\ \text { GE } & \text { Gradient Echo } \\ \text { GM } & \text { Gray Matter } \\ \text { GPU } & \text { Graphics Processing Unit } \\ \text { MR } & \text { Magnetic Resonance } \\ \text { MRI } & \text { Magnetic Resonance Imaging } \\ \text { NMR } & \text { Nuclear Magnetic Resonance } \\ \text { PD } & \text { Proton Density } \\ \text { PFG } & \text { Pulsed Field Gradient } \\ \text { RF } & \text { Radio Frequency } \\ \text { SE } & \text { Spin Echo } \\ \text { SBRT } & \text { Stereotactic Body Radiation Therapy } \\ \text { SNR } & \text { Signal to Noise Ratio } \\ \text { SQP } & \text { Sequential Quadratic Programming } \\ \text { SRS } & \text { Stereotactic Radiosurgery } \\ \text { SVM } & \text { Support Vector Machine } \\ \text { WM } & \text { White Matter }\end{array}$




\section{Radiotherapy}

"The dose makes the poison"

Paracelsus (1493-1541)

Radiotherapy is the therapeutic use of ionizing radiation, often with the intent to kill a tumor while minimizing damage to healthy surrounding tissue.

In 2003, the Swedish Council on Technology Assessment in Health Care (SBU) evaluated the role of radiotherapy in the treatment of tumors [56]. It was concluded that radiotherapy has an important role in the cure and palliation of many cancer types - contributing to cure in about $40 \%$ of the patients. Radiotherapy is also a highly cost-effective treatment [50]. In the future, the rapid technological developments of the entire radiotherapy process is expected to further increase its importance [63, 64].

This chapter provides a general background on radiotherapy: from the principles of how ionizing radiation interacts with biological matter to how treatment plans are designed.

\subsection{Radiobiology}

This chapter will provide an overview of how ionizing radiation interacts with living tissue, a field called radiobiology. The presentation that will follow covers the predominant view in radiobiology, although it is an active field of research where studies emphasizing different biological mechanisms have received significant attention $[22,55]$.

\subsubsection{Cellular damage caused by ionizing radiation}

Ionizing radiation primarily affects the cell by causing single- or doublestrand breaks in its DNA, see figure 2.1. A single-strand break is when 


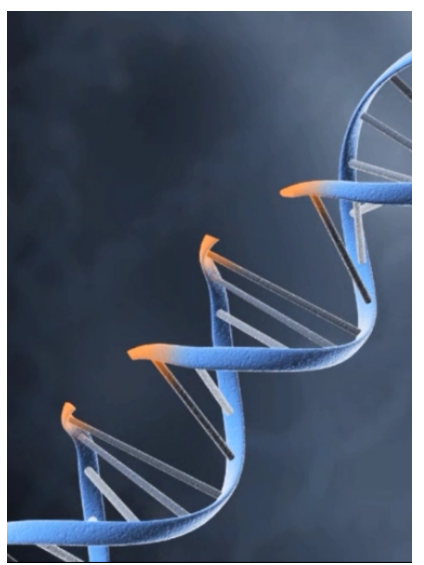

(a)

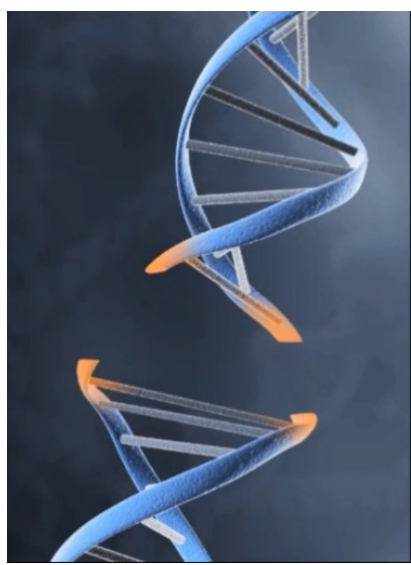

(b)

Figure 2.1: Ionizing radiation causes single- and double-strand breaks in the DNA. C)Elekta

one strand of the DNA-helix is destroyed. Since the opposing strand can be used as a template, single-strand breaks are easy to repair. This is not possibly with double-strand breaks, which are more difficult to repair. You can picture this as if the DNA-helix was a railway: a missing piece on one side and a train may still pass, but if both sides are missing you're in for a bumpy ride.

Failure to repair DNA damage may stop the cell's normal function and proliferation capacity, although it can take months until cell death actually occurs.

Different types of radiation interact with matter in different ways. A heavy charged particle moves in a straight track with dense interactions, while a photon's interactions are sparse and often scatters the photon in different directions. Because of their large mass and electric charge, protons and heavier ions are much more likely to cause a double-strand break compared with the sparsely ionizing photon, and therefore ions are more efficient in killing cells than photons, as shown in figure 2.2. The larger the mass of the ion, the more efficiently cells are killed.

Although radiation energy is deposited in discrete events on a microscopic level, it is convenient to describe it using the local average of the energy depositions on a macroscopic scale. This is done with the quantity absorbed dose - or simply dose - that describes the average energy absorbed by a mass of e.g. tissue or water. Absorbed dose is expressed in the unit of Gray (Gy). One Gy is the absorption of one Joule $(\mathrm{J})$ per kilogram $(\mathrm{kg})$ of mass. 


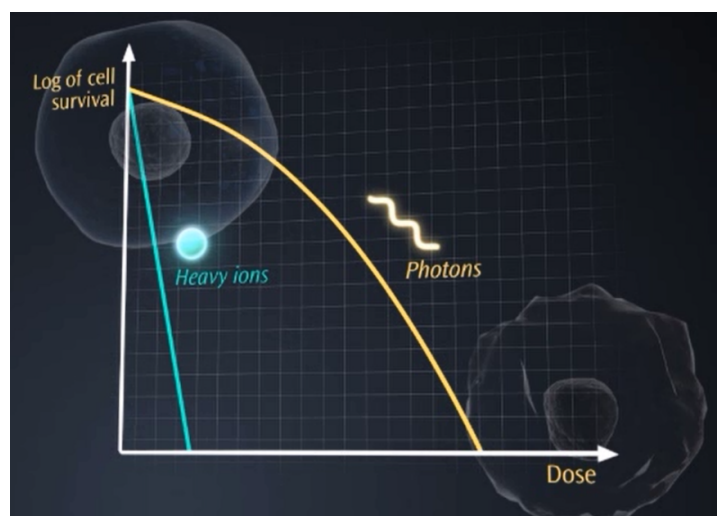

Figure 2.2: The large mass and electric charge of heavy ions make them more efficient at killing cells than photons. CElekta

Gamma radiation is another name for highly energetic photons. As a rule of thumb, one third of the DNA damage caused by gamma radiation is from direct photon interaction, whereas the remaining two-thirds of the damage is caused by free radicals induced by the radiation.

In the case of gamma radiation, one Gy of absorbed dose in tissue will cause approximately 1000 single-strand breaks, 40 double-strand breaks and a large amount of other aberrations in the DNA [72]. Damage in the double helix of the DNA may at subsequent cell divisions lead to cell death.

\subsubsection{The five $R$ 's of radiobiology}

Almost a century of research on the biological basis of radiotherapy has revealed five factors that are critical in determining the net effect of radiotherapy on tumors $[10,12,61,75]$. They are as follows:

- Repair - the ability to repair sublethal cellular damage varies between cell types.

- Redistribution - cells are more or less radiosensitive depending on the phase of their cell cycles.

- Repopulation - cells proliferate over time.

- Reoxygenation - presence of oxygen increases radiosensitivity.

- Radiosensitivity - different cell types have different intrinsic radiosensitivity. 


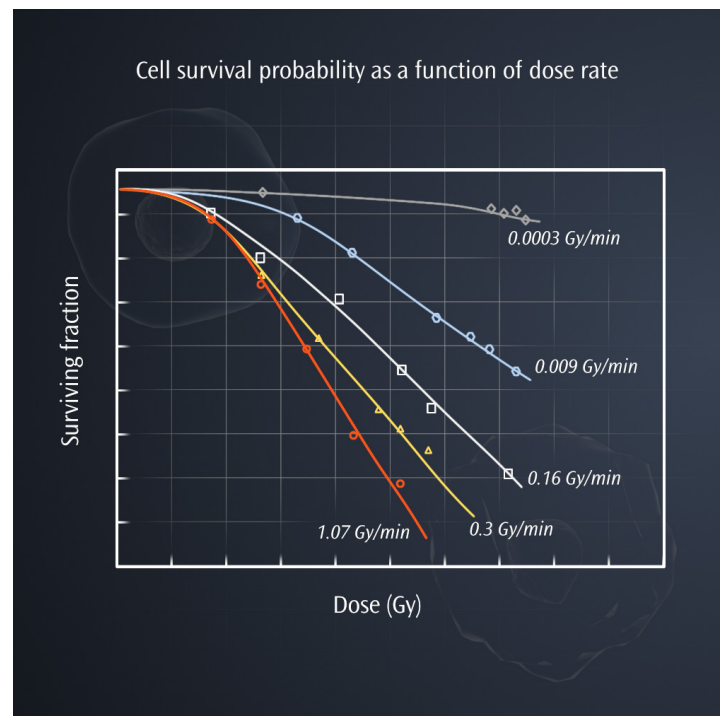

Figure 2.3: The effectiveness of the cell's DNA repair system means that the probability of killing cells reduces as the dose rate decreases. (C)Elekta

Each of these effects can work both ways. For example, if a given dose of radiation is divided into a number of fractions, then redistribution and reoxygenation may over time redistribute surviving tumor cells into more sensitive states, increasing overall cell kill. On the other hand, because of repair and repopulation, cells recover and proliferate, increasing overall cell survival. Modern radiotherapy strives to manipulate these effects to maximize tumor cell kill while avoiding normal tissue toxicities.

We will now survey each of these effects in some more detail.

\section{Repair}

Our DNA constantly suffers damage from both internal and external factors. As a consequence, DNA repair processes are always active. Their effectiveness means that if the dose rate (dose per unit time) decreases, the total dose required to achieve the same probability of cell kill increases. This is illustrated in figure 2.3. The size of the effect depends on the cell type, but tumor cells are often less efficient at repairing than normal cells.

Repair processes are particularly relevant if the dose rate is low, the irradiation time is long or if the treatment is spread out over time. 


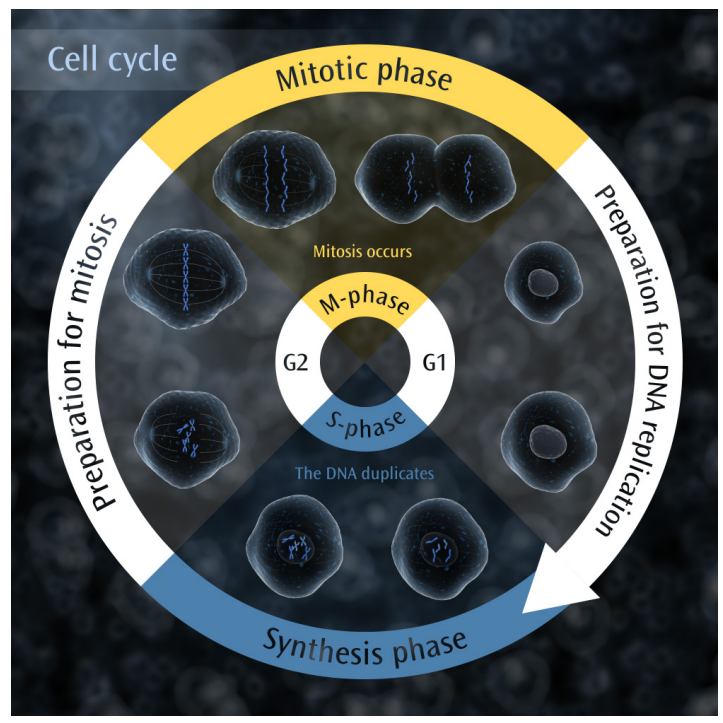

Figure 2.4: The life cycle of a dividing cell consists of four phases. (C)Elekta

\section{Redistribution}

The life cycle of a dividing cell consists of four phases [5], shown in figure 2.4 :

- G1 - preparation for DNA replication

- $\mathrm{S}$ - synthesis, DNA is replicated

- G2 - preparation for mitosis

- M - mitosis (cell division)

The sensitivity to radiation varies with phases of the cell cycle. As can be seen in figure 2.5, the cell is most resistant to radiation during DNA replication and most sensitive during mitosis.

By fractionating the treatment, i.e. dividing the total dose into a number of fractions spread out over time, tumor cells that were in a relatively radioresistant phase of the cell cycle during one fraction may cycle into a sensitive phase of the cycle before the next fraction is given.

\section{Repopulation}

Cells proliferate over time. However, the rate of proliferation varies widely depending on the cell type. In particular, cancer is characterized by uncon- 


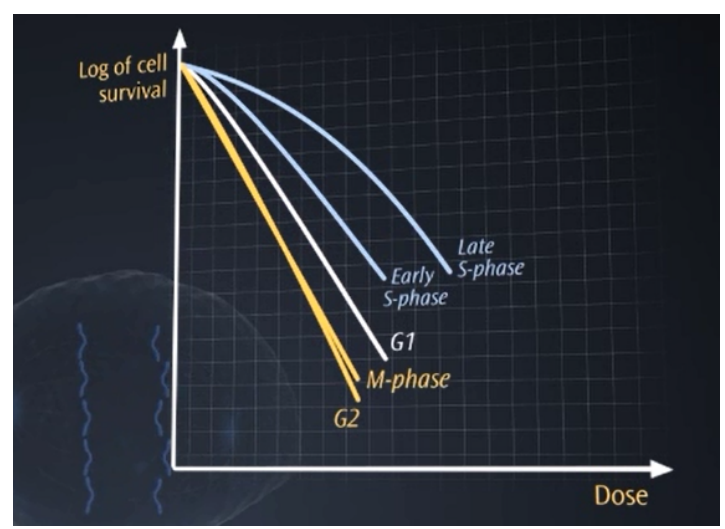

Figure 2.5: A cell's sensitivity to radiation depends on its phase in the cell cycle. (C)Elekta

trolled cell growth. If a treatment is fractionated there is a risk that the tumor repopulates.

\section{Reoxygenation}

To sustain its rapid growth, a tumor requires more oxygen (and nutrients) than healthy tissue and - although promoting blood vessel growth [29, 30]often outgrows its blood supply, leaving portions of the tumor with regions where the oxygen concentration is significantly lower than in healthy tissues. Tumor hypoxia is when tumor cells are deprived of oxygen.

The presence of molecular oxygen increases DNA damage through the formation of oxygen free radicals [28]. Moreover, hypoxia induces proteome and genome changes that may have a substantial impact on radioresistance $[32,70]$. Because of these effects, about three times as large radiation dose is required to achieve the same level of cell kill under hypoxic conditions as under normal conditions [23].

Even when only a small part of tumor is hypoxic, the effect of radiation is impaired. This is illustrated in figure 2.6, that shows the surviving fraction of cells as a function of dose in a mixed population of cells of different hypoxic content.

When radiosensitive well-oxygenated tumor cells die as a result of irradiation, oxygen becomes available to the hypoxic cells. This mechanism can be taken advantage of by fractionating the treatment. 


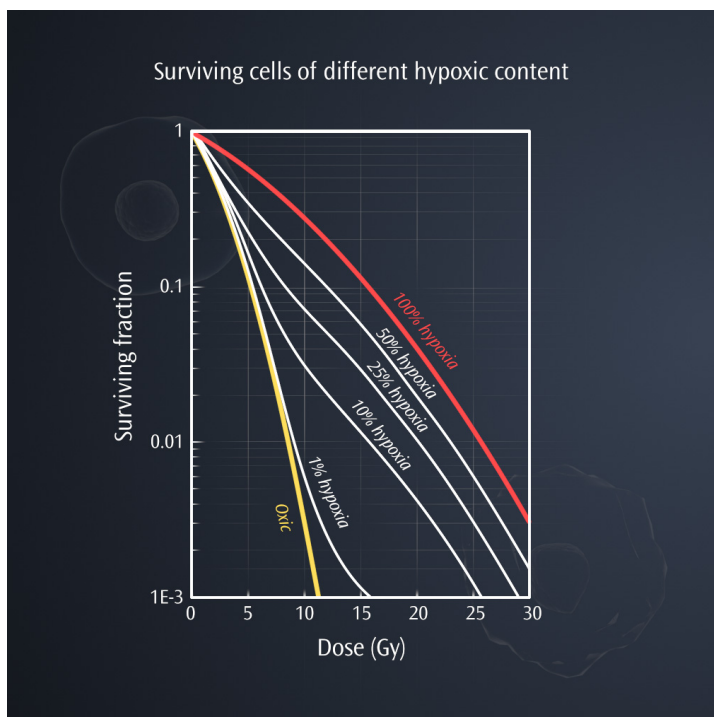

Figure 2.6: Regions in the tumor deprived of molecular oxygen (hypoxia) makes it more radioresistant. (C)Elekta

\section{Radiosensitivity}

There is an inherent difference in radiosensitivity between different cell types $[16,19]$. The most sensitive cells are those that are undifferentiated (stem cells), well nourished, dividing quickly and highly active metabolically. Although not universally true, tumor cells are more radiosensitive than the majority of body cells.

\section{$2.2 \quad$ Radiotherapy modalities}

Radiotherapy can be carried out with a radiation source placed either inside the body (brachytherapy) or outside the body (external beam radiotherapy).

In brachytherapy, radioactive sources are placed temporarily or permanently. Temporary sources are usually placed with the help of a hollow tube, called an applicator, positioned close to or inside the target.

In external beam radiotherapy, the patient typically lies on a couch while an external source directs an energetic beam at the target. External beam radiotherapy with gamma radiation (photons) is, by far, the most common type of radiotherapy. In particle therapy, another form of external beam 


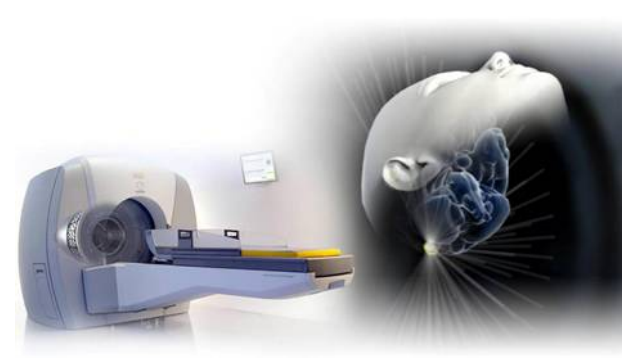

Figure 2.7: The Leksell Gamma Knife@is a machine for stereotactic radiosurgery that irradiates cerebral targets with narrow high-energy beams of gamma radiation from many directions. (C)Elekta

radiotherapy, beams of energetic protons, neutrons or heavier ions are used.

Stereotactic treatments refer to external beam radiotherapy where large doses are delivered in a few fractions with exceptionally high targeting accuracy. A distinction is often made between Stereotactic radiosurgery (SRS), where the target is in the brain or spine, and stereotactic body radiation therapy (SBRT) where the target is elsewhere in the body.

This thesis was written mainly with applications to stereotactic radiosurgery in mind.

\subsubsection{Stereotactic radiosurgery}

In 1951, the concept of radiosurgery was introduced by the Swedish neurosurgeon Lars Leksell as A "single high dose fraction of radiation, stereotactically directed to an intracranial region of interest" [42]. Although its scope has broadened over the years, the crucial role of precise targeting in stereotactic radiosurgery remains the same.

The heritage of Lars Leksell lives on in the Leksell Gamma Knifeß), a machine for SRS that irradiates cerebral targets with narrow high-energy beams of gamma radiation from many directions, see figure 2.7. At the beams intersection, energy from all beams is delivered to the cells. Outside that region, the radiation dose decreases rapidly. This, together with the exceptional targeting accuracy of such a SRS system, enables the delivery of a large, and yet localized, dose to the target while minimizing dose to the surrounding normal tissue.

Although there is strong clinical evidence for the effectiveness of stereotactic radiosurgery $[6,44,45,46]$, its radiobiological effects are not completely understood. There's an ongoing debate whether additional effects to the 


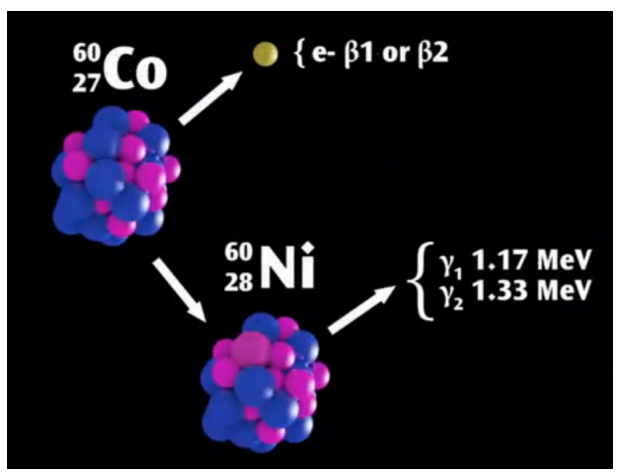

Figure 2.8: Radioactive decays of Cobalt-60, which is used in the Gamma Knife. (C)Elekta

ones described in section 2.1.2 come into play for SRS and SBRT [9, 12, 38, $53]$.

\subsection{Physics of Cobalt-60 radiation}

In the Gamma Knife, radiation is produced by the radioactive decay of Cobalt-60 $\left({ }^{60} \mathrm{Co}\right)$ sources. Radioactivity is the process whereby unstable atoms release their energy, generally by emitting massive particles or photons, in order to reach its stable, non-radioactive, state. This transition from an unstable atom to the final stable state can include several steps. At each such step energy is radiated.

Figure 2.8 depicts how ${ }^{60}$ Co releases its excess energy. One of the neutrons of the ${ }^{60} \mathrm{Co}$ nucleus transforms, through weak interaction, into a proton and an electron. The electron is instantly emitted by the nucleus, but never reaches the patient since it is absorbed by matter in the radiation source. By this process the ${ }^{60} \mathrm{Co}$ nucleus transforms into a new element, Nickel$60\left({ }^{60} \mathrm{Ni}\right)$. The nucleus of ${ }^{60} \mathrm{Ni}$ instantly emits two gamma photons with energies of 1.17 and $1.33 \mathrm{MeV}$, respectively.

Each radionuclide has a characteristic half-life. The half-life of ${ }^{60} \mathrm{Co}$ is 5.27 years. In practice this means that after little more than five years the radioactivity is only half of the initial radioactivity and the irradiation time required to achieve a certain dose is doubled. Treatment times are prolonged accordingly, meaning that fewer patients can be treated per day, thus sources need to be replaced. 


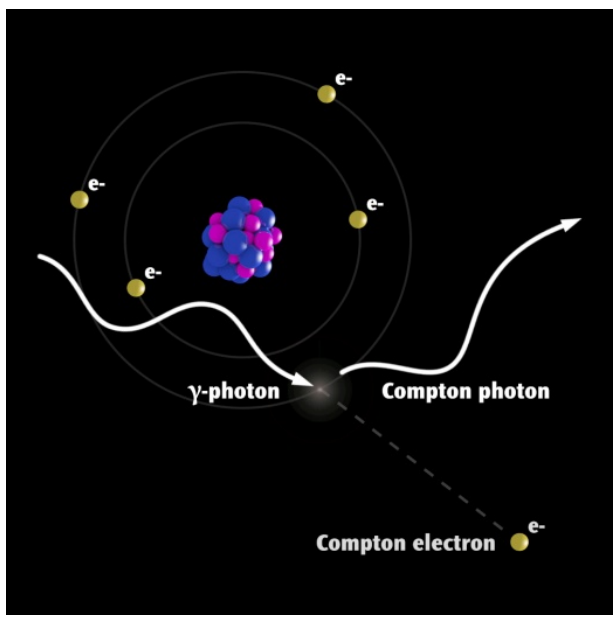

Figure 2.9: Gamma radiation used in external beam radiotherapy mainly interacts with tissue via Compton scattering, a process where the incoming photon scatters inelastically against an electron. CElekta

\subsubsection{Energy deposition in photon beams}

In conventional external beam radiotherapy as well as stereotactic radiosurgery with the Gamma Knife, the radiation consists of photons in the $\mathrm{MeV}$ range. In this energy range, the interaction with tissue occurs mainly via a process called Compton scattering, shown in figure 2.9. In short, Compton scattering is an interaction in which the incoming photon scatters inelastically against an electron. The energy imparted to the electron makes it recoil and it is thereby ejected from the atom. The remaining part of the energy is emitted as a scattered photon in a different direction than the electron, such that the overall momentum is conserved.

The recoiling electron ionizes and excites atoms along its trajectory until its kinetic energy is lost. Since the mean free path of the electron is significantly shorter than that of the scattered photon, its energy is deposited far more locally. Therefore it is common to make a distinction between primary dose, deposited by the electron close to the place of interaction, and secondary dose due to the scattered photon, which also eventually gives of its energy by electron interactions. 


\subsection{Treatment planning}

In radiotherapy, treatment planning is the - almost entirely computer basedprocess in which a team of medical personnel develop a patient-specific radiotherapy treatment plan. Typically, medical images acquired with Computed Tomography (CT) or magnetic resonance imaging (MRI) are used to create a patient model in which additional information such as the treatment target and the spatial distribution of the dose is overlaid.

Broadly speaking, treatment planning consists of two main steps: deciding what to treat and how to treat it. The target, and organs at risk, are specified by delineating them on a primary set of images. Often supplementary sets of images are also used. Next, a decision is made on the desired dose to the target and possibly also what doses that can be tolerated in the organs at risk. The clinician then attempts to realize this plan, either by manually specifying the machine parameters (forward planning) or using an optimization procedure (inverse planning). This process is interlaced with simulations of how the dose will be deposited given the current machine parameters.

Today, CT is the mainstay of the radiotherapy workflow [65]. However, the superior soft tissue contrast of MRI already makes it the preferred modality for a number of anatomical locations such as the brain, abdomen and pelvis. This is why stereotactic radiosurgery - contrary to general radiotherapyhas a tradition of planning primarily on MR images. There is currently a strong movement towards MR based radiotherapy [65], fueled also by the possibility of using MRI for so called functional imaging, i.e. imaging physiology instead of anatomy.

One hurdle that an entirely MR based workflow has to overcome-and which is the motivation of papers I and II-is how to do dose calculations when a CT is not available. To understand why this is an issue, the next section describes how CT based dose calculations are done.

\subsubsection{Dose calculations}

The dose quantifies the radiation energy delivered to the tissue. As described in section 2.1.2, the dose directly relates to the survival probability of irradiated cells and so plays a key role in treatment planning. The spatial distribution of the dose, given a set of machine parameters, can be estimated by a range of different algorithms. The choice of algorithm involves a tradeoff between computational speed and accuracy. The simplest (and fastest) dose calculation algorithms approximate all tissue as water-a reasonably good approximation for soft tissue in homogeneous regions [15, 54] but less so in heterogeneous regions [76]. 
To correct for tissue heterogeneity, more accurate dose calculation algorithms use the Hounsfield units (HU) provided by CT data. As described in section 2.3, the incoming photons mainly interact with electrons in the tissue atoms through Compton scattering. In the energy range of radiotherapy, the linear attenuation coefficient for Compton scattering is almost completely dependent on the electron density $[20,59]$. For MR, on the other hand, there is no relation between image values and electron density.

Hounsfield units have two calibration points, water and air, which are set to 0 and $-1000 \mathrm{HU}$. Conversion from arbitrary Hounsfield units to electron density can be done using semi-empirical formulas [20,37] or via a tissue look up table $[33,34]$. Proton and ion beams interact with tissue in a variety of ways, each with a different relationship to the material characteristics obtained from CT [47]. This implies that for protons and ions, the ability to precisely define tissues based on $\mathrm{CT}$ scans has a significant impact on the accuracy of dose calculations $[36,58]$. For MR, on the other hand, there is no relation between image values and electron density.

There are two major types of dose calculation algorithms that take tissue heterogeneity into account: superposition-based [2] and Monte Carlo-based [60]. In all of the approaches using heterogeneity corrections, the computationally intense part is simulation of the electron transport.

Superposition-based algorithms convolve the energy released by a photon with pre-computed energy deposition kernels that are scaled with density. In a pencil beam algorithm $[4,39]$, the precalculated dose distribution from a single ray of photons in water is scaled with the density distribution along the ray, disregarding lateral variations. Collapsed cone algorithms [3] also take lateral variations into account and are thus more accurate.

Monte Carlo methods are stochastic methods that explicitly simulate the transport of a large number of particles, successively building up an accurate estimate of the dose distribution. Monte Carlo methods are considered the gold standard for dose calculations in radiotherapy [20,37]. Their computation complexity is, however, an obstacle to the routine use of Monte Carlo in a clinical setting, although significant advances have been made by using variance reduction techniques and taking advantage of the graphical processing unit (GPU) to speed up computations [31]. 


\section{Magnetic resonance imaging}

"Mathematics is not a spectator sport"

George M. Phillips (1938-)

Since its introduction in the 1970s, magnetic resonance imaging (MRI) has evolved into an indispensable tool for medical imaging. Its excellent soft tissue contrast and inherent patient safety [21] makes MRI preferable to other modalities, such as computed tomography (CT), for a range of imaging tasks. This is certainly true for many diagnostic applications, but MRI also has a prominent role in radiotherapy planning [65].

Clinical MR scanners create a magnetic field with typical strengths of 1.5 or 3 Tesla (T) - about 50000 times stronger than Earth's magnetic fieldto amplify the effect of a quantum mechanical phenomena known as nuclear magnetic resonance (NMR). It is based on the fact that protons and neutrons, that make up every atomic nucleus, have an intrinsic quantum property called spin. These spins align either parallel or anti-parallel with an external magnetic field. There is a slight energy difference between the two states - corresponding to the energy of a radio frequency (RF) photon. This implies that radio frequency emitters and receivers can be used to probe the image subject, and measure the strength of the re-emitted signal from different areas. Among biologically relevant elements, this effect is by far easiest to make use of for hydrogen, as it is both the most abundant and the one with strongest interaction with an externally applied magnetic field.

Since nuclear magnetic resonance is an intrinsically quantum mechanical phenomena, a thorough description of the signal origin requires a quantum mechanical perspective $[1,13,26]$. Such a description is given in the following section, readers unfamiliar with quantum mechanics may instead refer to texts providing a classical treatment (which is perhaps more intuitive but can be misleading) [43, 49]. The rest of the chapter then describes how the magnetic resonance phenomena can be utilized to generate images with 
various contrasts.

\subsection{The origin of the MR signal}

Nuclear magnetic resonance is based on a quantum mechanical property called spin: an intrinsic angular momentum carried by elementary particlesof which the proton is of primary interest. The proton has spin $1 / 2$, which means that measuring, say, the $z$ component of its spin angular momentum $\mathbf{S}$ can return either plus or minus $\frac{1}{2} \hbar$ ('spin up' or 'spin down'), where $\hbar$ is Planck's constant divided by $2 \pi$. The spin is directly tied to the magnetic moment $\boldsymbol{\mu}$ of the particle. For a proton at rest, the relation is given by

$$
\boldsymbol{\mu}=\gamma \mathbf{S}
$$

where $\gamma=42.58 \mathrm{MHz} / \mathrm{T}$ is the gyromagnetic ratio. In general, the gyromagnetic ratio is different for different particles. The existence of a magnetic moment means that the particle will be affected by an external magnetic field. More precisely, the potential energy $E$ of a proton in an external magnetic field $\mathbf{B}=B \hat{\mathbf{z}}$ is

$$
E=-\boldsymbol{\mu} \cdot \mathbf{B}
$$

The corresponding Hamiltonian operator can, in matrix form, be written as

$$
H=-\gamma B S_{z}=\left(\begin{array}{cc}
-\frac{1}{2} \hbar \omega_{0} & 0 \\
0 & \frac{1}{2} \hbar \omega_{0}
\end{array}\right) .
$$

The quantity $\omega_{0}=\gamma B$ is called the Larmor frequency, for a reason soon to be explained. It follows by inspection that the Hamiltonian (3.3) has the eigenstates

$$
\boldsymbol{\psi}_{\uparrow}=\left(\begin{array}{l}
1 \\
0
\end{array}\right), \quad \boldsymbol{\psi}_{\downarrow}=\left(\begin{array}{l}
0 \\
1
\end{array}\right),
$$

with the corresponding eigenvalues

$$
E_{\uparrow}=-\frac{1}{2} \hbar \omega_{0}, \quad E_{\downarrow}=\frac{1}{2} \hbar \omega_{0},
$$

which shows that the energy is lowest when the magnetic moment is aligned with the external field. This splitting of the energy levels is known as the Zeeman effect.

The time-evolution of the magnetic moment follows from the time-dependent Schrödinger equation [25],

$$
i \hbar \frac{\partial \boldsymbol{\psi}}{\partial t}=H \boldsymbol{\psi}
$$




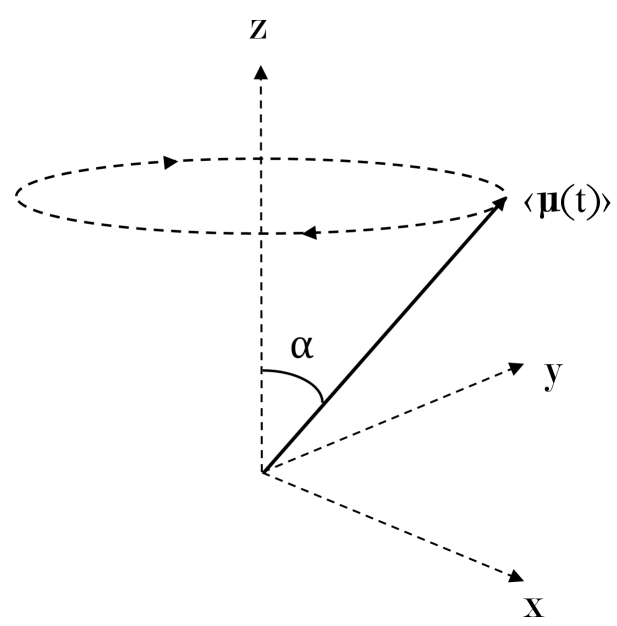

Figure 3.1: The expected value of the magnetic moment $\langle\boldsymbol{\mu}\rangle$ is tilted at a constant angle $\alpha$ to the direction of the external magnetic field and precesses about it with the Larmor frequency $\omega_{0}$.

Expanding $\boldsymbol{\psi}(t)$ using the eigenstates (3.4) gives

$$
\boldsymbol{\psi}(t)=c_{\uparrow} \boldsymbol{\psi}_{\uparrow} e^{-i E_{\uparrow} t / \hbar}+c_{\downarrow} \boldsymbol{\psi}_{\downarrow} e^{-i E_{\downarrow} t / \hbar}=\left(\begin{array}{c}
c_{\uparrow} e^{i \omega_{0} t / 2} \\
c_{\downarrow} e^{-i \omega_{0} t / 2}
\end{array}\right),
$$

where the constants $c_{\uparrow}$ and $c_{\downarrow}$ are determined by the initial conditions. From the normalization requirement, it follows that a natural way of rewriting these are as $c_{\uparrow}=\cos (\alpha / 2)$ and $c_{\downarrow}=\sin (\alpha / 2)$ for a fixed angle $\alpha$. For a spin $1 / 2$ particle $\alpha= \pm 54.44^{\circ}$ [43]. Since the magnetic moment $\boldsymbol{\mu}$ is related to $\mathbf{S}$ by a constant, we find that its expected value is

$$
\begin{aligned}
& \left\langle\mu_{x}\right\rangle=\gamma \boldsymbol{\psi}(t)^{\dagger} S_{x} \boldsymbol{\psi}(t)=\frac{\gamma \hbar}{2} \sin \alpha \cos \left(\omega_{0} t\right), \\
& \left\langle\mu_{y}\right\rangle=\gamma \boldsymbol{\psi}(t)^{\dagger} S_{y} \boldsymbol{\psi}(t)=-\frac{\gamma \hbar}{2} \sin \alpha \sin \left(\omega_{0} t\right), \\
& \left\langle\mu_{z}\right\rangle=\gamma \boldsymbol{\psi}(t)^{\dagger} S_{z} \boldsymbol{\psi}(t)=\frac{\gamma \hbar}{2} \cos \alpha .
\end{aligned}
$$

As illustrated in figure 3.1, this means that $\langle\boldsymbol{\mu}\rangle$ is tilted at a constant angle $\alpha$ to the direction of the external magnetic field and precesses about it with the Larmor frequency $\omega_{0}$.

From equation (3.2), we have that the energy difference between the spin up and spin down states is $\Delta E=\hbar \omega_{0}$. Now-according to the MaxwellBoltzmann theory in statistical mechanics - the probability of finding the 
system in a state with energy $\epsilon$ when in thermal equilibrium with a reservoir at temperature $T$ (body temperature) is given by

$$
p(\epsilon)=\frac{e^{-\epsilon / k_{B} T}}{Z},
$$

where the partition function $Z$ serves as a normalization factor and $k_{B}$ is Boltzmann's constant. Consequently, there is a larger probability of finding a proton in the low energy state, i.e. with spin parallel to the magnetic field (spin up).

At physiological temperatures and with a magnetic field of $1 \mathrm{~T}$, there are about 3 spins per million more in the low energy state. This might not seem much, but considering that the number of protons in a sample, $N$, is on the order of Avogadro's constant $\left(6.022 \cdot 10^{23}\right)$ it produces an observable magnetic moment.

The excess number of protons in the low energy state produces a net magnetization $\mathbf{M}=N\langle\boldsymbol{\mu}\rangle$. At equilibrium, it points in the direction of the magnetic field, referred to as the longitudinal magnetization $M_{z}$. The component of the magnetization orthogonal to the external magnetic field is called the transverse component; it is often expressed using the complex notation $M_{x y}=M_{x}+i M_{y}$. At equilibrium it is zero, as the spins are randomly oriented in the xy-plane and their magnetic effects therefore cancel each other.

\subsection{Excitation and relaxation}

In the previous section, we saw how nuclear magnetism can be used to produce a net magnetization in a sample. Creating an actual image is all about manipulating this net magnetization. This is where the resonance part of magnetic resonance imaging comes in; only photons with an energy that exactly matches the energy difference between the spin states can influence the spin. Such photons are typically in the radio frequency $(\mathrm{RF})$ range for magnetic fields on the order of $1 \mathrm{~T}$. To see how RF pulses affect the net magnetization, first consider a coordinate system that rotates about the $z$-axis at the Larmor frequency,

$$
\begin{aligned}
& \hat{x}^{\prime}=\hat{x} \cos \left(\omega_{0} t\right)-\hat{y} \sin \left(\omega_{0} t\right), \\
& \hat{y}^{\prime}=\hat{x} \sin \left(\omega_{0} t\right)+\hat{y} \cos \left(\omega_{0} t\right), \\
& \hat{z}^{\prime}=\hat{z} .
\end{aligned}
$$

Assuming that the radio frequency pulse is left circularly polarized and has a time-dependent magnetic field strength $B_{1}(t)$ its associated magnetic field can be written simply as $\mathbf{B}_{1}(t)=B_{1}(t) \hat{x}^{\prime}$. From a derivation similar to the 
one for the static case, one may show $[1,26]$ that the expected value of the magnetic moment after applying the pulse for a time $\tau$ are

$$
\begin{aligned}
\left\langle\mu_{x^{\prime}}(\tau)\right\rangle & =\left\langle\mu_{x^{\prime}}(0)\right\rangle \\
\left\langle\mu_{y^{\prime}}(\tau)\right\rangle & =\left\langle\mu_{y^{\prime}}(0)\right\rangle \cos \theta+\left\langle\mu_{z^{\prime}}(0)\right\rangle \sin \theta \\
\left\langle\mu_{z^{\prime}}(\tau)\right\rangle & =-\left\langle\mu_{y^{\prime}}(0)\right\rangle \sin \theta+\left\langle\mu_{z^{\prime}}(0)\right\rangle \cos \theta
\end{aligned}
$$

where we have introduced the flip angle

$$
\theta=\int_{0}^{\tau} \gamma B_{1}(t) d t
$$

Thus, the RF pulse rotates the magnetization about its axis (here $x^{\prime}$ ) with an angle given by the flip angle.

When the pulse is turned off the sample will start to relax to its equilibrium state. This happens at different time scales for the transverse and longitudinal magnetization. Longitudinal magnetization, with characteristic time $T_{1}$, is limited by how fast the excited spins release their energy to the surrounding lattice. Transverse relaxation, with characteristic time $T_{2}$, is the gradual loss of precessional coherence, in other words the precessional frequencies of the individual spins spread out over time. Both relaxation processes are usually ascribed to time-dependent microscopic fluctuations in the magnetic field arising from the ever-present thermal motion [1].

The relaxational effects are captured by the phenomenological Bloch equations which, in the rotating frame, can be written as

$$
\begin{aligned}
\frac{d M_{z^{\prime}}}{d t} & =-\frac{M_{z^{\prime}}-M_{z}^{0}}{T_{1}}, \\
\frac{d M_{x^{\prime} y^{\prime}}}{d t} & =-\frac{M_{x^{\prime} y^{\prime}}}{T_{2}},
\end{aligned}
$$

where $M_{z}^{0}$ is the longitudinal magnetization at thermal equilibrium. Equation (3.19) yields an exponential regrowth of the longitudinal magnetization, according to

$$
M_{z^{\prime}}(t)=M_{z}^{0}\left(1-e^{-t / T 1}\right)+M_{z^{\prime}}(0) e^{-t / T_{1}},
$$

where $M_{z^{\prime}}(0)$ is the longitudinal magnetization along the $z^{\prime}$-axis immediately after the RF pulse. Similarly, equation (3.20) results in an exponential decay of the transverse magnetization

$$
M_{x^{\prime} y^{\prime}}(t)=M_{x^{\prime} y^{\prime}}(0) e^{-t / T 2},
$$

where $M_{x^{\prime} y^{\prime}}(0)$ is the transverse magnetization immediately after the RF pulse. The precession of the transverse magnetization in the stationary 


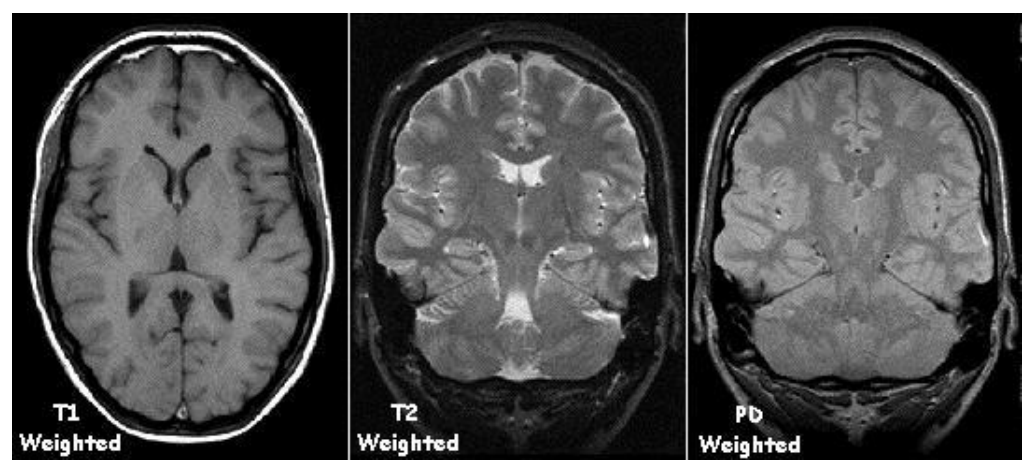

Figure 3.2: Examples of MR images with contrast primarily due to variations in $T_{1}, T_{2}$ or proton density (PD). The left image shows an axial section of a brain whereas the center and right ones show coronal sections.

\begin{tabular}{l|c|c|} 
Tissue type & $T_{1}(\mathrm{~ms})$ & $T_{2}(\mathrm{~ms})$ \\
\hline White matter (WM) & 600 & 80 \\
Gray matter (GM) & 950 & 100 \\
Cerebrospinal fluid (CSF) & 4500 & 2200 \\
Muscle & 900 & 50 \\
Fat & 250 & 60
\end{tabular}

Table 3.1: Typical values of $T_{1}$ and $T_{2}$ for some different tissue types in a magnetic field of strength $1.5 \mathrm{~T}$ [26].

coordinate frame, $M_{x y}=M_{x^{\prime} y^{\prime}} e^{-i \omega_{0} t}$, can be detected by an antenna coil via induction. This is what constitutes the signal in MRI!

The contrast in MR images stems from the tissue dependence of the proton density and the relaxation times $T_{1}$ and $T_{2}$. Scans that primarily achieve contrast from differences in $T_{1}$, called $T_{1}$-weighted scans, achieve a strong signal from fat, gray matter and white matter, whereas the signal from the cerebrospinal fluid (CSF) is weak. $T_{2}$-weighted scans, on the other hand, display a strong response from CSF and intermediate response from fat, gray matter and white matter. Figure 3.2 illustrates the different appearances of $T_{1^{-}}, T_{2^{-}}$and proton density $(\mathrm{PD})$ weighted MR images. Some typical values of the relaxation times are shown in table (3.1). Bone has an extremely short $T_{2}$ relaxation time, $0.4-0.5 \mathrm{~ms}$, which makes it technically difficult-but not impossible - to measure [57, 73, 74]. Because of the short relaxation time, conventional MRI sequences result in a very weak response from bone, thus making it difficult to distinguish from air (which has a low signal because of its low proton density). For applications in radiotherapy, this can be troublesome since bone is the material which attenuates radiation the most 
and air barely attenuates it at all.

The presence of inhomogeneities in the external magnetic field can accelerate the transverse relaxation, making its effective characteristic time $T_{2}^{*}$ machine-dependent. The sequence in which RF pulses and magnetic field gradients are applied makes the signal dependent on either $T_{2}$ or $T_{2}^{*}$. This is the subject of the next section.

\subsection{Pulse sequences}

There are three basic contrast mechanisms in MRI: $T_{1}$ relaxation, $T_{2}$ relaxation and proton density (PD). Although it is possible to apply an RF pulse and simply measure the signal as the magnetization returns to equilibrium, referred to as Free Induction Decay (FID), this is not what is typically done. As alluded to in the previous section, a clever application of RF pulses and magnetic field gradients makes it possible to accentuate a contrast mechanism of choice.

This section will first describe the gradient system of an MR scanner, which is crucial both for contrast selectivity and, as will be shown later, for spatial encoding of the signal. Then, we will describe two fundamental pulse sequences, the spin echo and the gradient echo, and look at how different pulse parameters result in images with different contrast.

\subsubsection{Gradients}

The system generating magnetic field gradients usually consists of three orthogonal gradient coils that generate a time-varying magnetic field $\mathbf{B}(\mathbf{r}, t)=$ $\left(B_{x}, B_{y}, B_{z}\right)$. Because of the much stronger static magnetic field $\mathbf{B}_{0}=B_{0} \hat{z}$, only the part of $\mathbf{B}$ parallel with the $\hat{z}$-axis will make a significant contribution to the total magnetic field [13]. Moreover, the gradients are designed to generate a magnetic field that varies linearly with the position $\mathbf{r}$. This means that it is usually sufficient to describe them by $\mathbf{G}(t)=\nabla_{\mathbf{r}} B_{z}(\mathbf{r}, t)$.

The usefulness of a spatially varying magnetic field comes from the fact that the Larmor frequency, $\omega=\gamma B$, depends on the local magnitude of the magnetic field. Thus, a spatially varying magnetic field implies a spatially varying frequency

$$
\omega(\mathbf{r})=\gamma\left(B_{0}+\mathbf{r} \cdot \mathbf{G}\right) .
$$

Important specifications for a gradient system include the maximum gradient strength and the rate at which the gradient strength can be changed, referred to as the slew rate. Today's clinical scanners have a maximum gradient strength on the order of $50 \mathrm{mT} / \mathrm{m}$ (millitesla per meter) and a slew rate on the order of $100 \mathrm{mT} / \mathrm{m} / \mathrm{s}$. Depending on the application, these 
specifications may have a strong impact on image quality and/or acquisition time. This was one of the driving factors for the pulse sequence optimization described in paper III.

\subsubsection{Echoes}

Echo sequences consists of two equally long parts: a dephasing part and a refocusing part. The two fundamental types of echo sequences, spin echo (SE) and gradient echo (GE), differ in how this dephasing and refocusing is achieved. Both, however, begin with an RF excitation pulse. A spin echo always uses a $90^{\circ}$ flip angle whereas the flip angle can be varied in a gradient echo. Spin echoes produce images of higher quality but are less flexible than gradient echoes.

\section{Spin echo}

To create a spin echo, the spins are allowed to dephase naturally after the $90^{\circ}$ pulse. After a time $\tau$, a $180^{\circ} \mathrm{RF}$ pulse is applied, reversing the phase angles. An echo is formed when the spins are back in phase again, which happens at the echo time $T_{E}=2 \tau$. This phase reversal trick removes the effects of any magnetic field inhomogeneities, so the echo height will only depend on $T_{2}\left(\right.$ not $\left.T_{2}^{*}\right)$.

\section{Gradient echo}

To create a gradient echo, a gradient is first applied during a time interval $\tau$ immediately following the excitation pulse. This causes rapid dephasing of the spins. Then the opposite gradient is applied, which rephases the spins. As before, the spins are back in phase again at the echo time $T_{E}=$ $2 \tau$, forming an echo. Contrary to spin echoes, gradient echos do not fully compensate for the inhomogeneity of the magnetic field, so the echo height depends on $T_{2}^{*}$. On the other hand, gradient echoes are more flexible since it is possible to vary the flip angle.

\subsubsection{Pulse parameters}

To increase the signal-to-noise ratio (SNR) of MR images, it is common to repeat the same sequence a number of times and average the results. To retain signal strength, however, the longitudinal magnetization must be allowed to recover between repetitions. This places a restriction on the number of times a sequence can be repeated during a given time interval. It is possible to use a repetition time $T_{R}$ such that the longitudinal magnetization only recovers partially between repetitions. After a while the same, steady-state, magnetization will be reached immediately before the next 
repetition starts. From the Bloch equations, (3.19) and (3.20), it is possible to show that, when using a flip angle $\theta$, the steady-state magnetization is

$$
\begin{aligned}
M_{z^{\prime}}^{s s} & =M_{z}^{0} \frac{\left(1-e^{-T_{R} / T_{1}}\right)}{1-\cos (\theta) e^{-T_{R} / T_{1}}}, \\
M_{x^{\prime} y^{\prime}}^{s s} & =M_{z}^{0} \frac{\left(1-e^{-T_{R} / T_{1}}\right)}{1-\cos (\theta) e^{-T_{R} / T_{1}}} \sin (\theta) e^{-T_{E} / T_{2}^{*}} .
\end{aligned}
$$

For a spin echo, these expressions can be simplified since $\theta=90^{\circ}$. Also, $T_{2}^{*}$ is then replaced by $T_{2}$. We see that the repetition time is related to the $T_{1}$ relaxation and the echo time is related to the $T_{2}$ relaxation.

\subsection{Signal localization}

If we were to apply a pulse sequence as described up to this point, we would get back the combined signal from every spin in the sample - that is not an image! To obtain an image it is necessary to encode where in the sample a signal comes from. This can be achieved with the help of the magnetic field gradients G. Signal localization involves two main steps: selective excitation and spatial encoding.

\section{Selective excitation}

In order to only excite a selected part of the volume, a gradient is applied during the RF pulse. Recall that this creates a spatially varying precession frequency,

$$
\omega(\mathbf{r})=\gamma\left(B_{0}+\mathbf{r} \cdot \mathbf{G}\right) .
$$

Only the protons at spatial locations where the precession frequency matches the RF frequency will be excited.

\section{Spatial encoding}

After a part of the volume has been excited, spatial information can be encoded into a signal during the free precession period. To see how, we first introduce what is called the $\mathbf{k}$-space formalism. A spin located at position $\mathbf{r}$ subjected to a time-varying gradient $\mathbf{G}$ will, in the rotating frame, acquire the phase

$$
\Phi(\mathbf{r}, t)=\int_{0}^{t} \gamma \mathbf{r} \cdot \mathbf{G}\left(t^{\prime}\right) d t^{\prime}=\mathbf{r} \cdot \gamma \int_{0}^{t} \mathbf{G}\left(t^{\prime}\right) d t^{\prime}=\mathbf{r} \cdot \mathbf{k},
$$

where we have introduced

$$
\mathbf{k}=\gamma \int_{0}^{t} \mathbf{G}\left(t^{\prime}\right) d t^{\prime}
$$



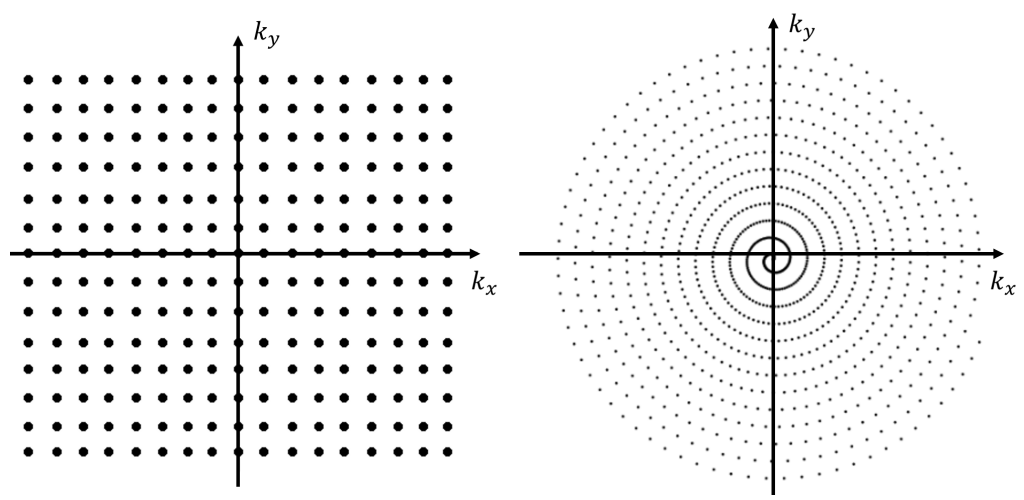

Figure 3.3: Sampling of k-space can be performed in different ways. The left image shows a 2D Cartesian sampling pattern and the right image shows a 2D spiral sampling pattern. Image adapted, with permission, from [18].

We also introduce the local normalized spin density

$$
\rho(\mathbf{r})=\frac{M_{x y}(\mathbf{r}, 0)}{\int M_{x y}(\mathbf{r}, 0) d \mathbf{r}} .
$$

The total normalized signal $S(t)$ can then be expressed as

$$
S(t)=\int \frac{M_{x y}(\mathbf{r}, t)}{M_{x y}(\mathbf{r}, 0)} d \mathbf{r}=e^{-t / T_{2}} \int \rho(\mathbf{r}) e^{-i \mathbf{k} \cdot \mathbf{r}} d \mathbf{r} .
$$

For clarity, we will omit the factor $e^{-t / T_{2}}$ in what follows, so that equation (3.30) can be written

$$
S(\mathbf{k})=\int \rho(\mathbf{r}) e^{-i \mathbf{k} \cdot \mathbf{r}} d \mathbf{r} .
$$

This shows that what we measure is really the Fourier transform of the normalized spin density. To create an image of $\rho(\mathbf{r})$ one therefore has to sample the signal $S(\mathbf{k})$ in $\mathbf{k}$-space. As shown in figure 3.3, this can be done in different ways. The easiest way is to sample on a Cartesian grid, as the image can then be reconstructed using the inverse Fourier transform

$$
\rho(\mathbf{r})=(2 \pi)^{-d / 2} \int S(\mathbf{k}) e^{i \mathbf{k} \cdot \mathbf{r}} d \mathbf{k},
$$

where $d$ is the dimension. The traversal of $\mathbf{k}$-space can be done using either frequency encoding and/or phase encoding. Frequency encoding involves sampling the signal at successive intervals in the presence of a read-out gradient. This corresponds to moving along a continuous trajectory in $\mathbf{k}$-space. 
Phase encoding is achieved by first applying a gradient for a time-interval, during which the spins acquire a phase according to equation (3.27), and then performing a measurement. This corresponds to doing a jump in $\mathbf{k}$ space. 



\section{L \\ Diffusion MRI}

"People have now-a-days got a strange opinion that everything should be taught by lectures. Now, I cannot see that lectures can do so much as reading the books from which the lectures are taken."

Dr. Samuel Johnson (1709-1784)

That diffusion would affect nuclear magnetic resonance measurements was realized already in 1950, when the idea of spin echoes was first proposed [27]. This is possible because nuclear spins have a phase that is determined by the history of the magnetic field they have experienced. Conversely, by manipulating the applied magnetic field, diffusion MRI (dMRI) serves as a probe of molecular motion. An interesting feature of diffusion MRI is that the scale of what is measured is determined by how far the molecules diffuse during an experiment - not by the resolution in the reconstructed image. In a typical dMRI experiment, the characteristic diffusion length is in the micrometer range, same as the order of cell sizes. This is why diffusion MRI is sometimes referred to as microstructure imaging.

This chapter covers the underlying principles of molecular translational motion and outlines how magnetic resonance can reveal those dynamics.

\subsection{Diffusion}

The internal energy of a substance is stored in the molecular motion of its constituent particles. We call this heat, and measure it using temperature. Under normal circumstances-when the temperature is not near absolute zero-a particle chosen at random will almost surely be on the move. A common misconception is that this particle's motion is random. It is not. It follows the reversible laws of mechanics, which means that reversing time would still produce a physically allowable motion. On the other hand, the particle density in a liquid substance makes molecular collisions inevitable. 
So, if you sample the particle's position at a time scale much larger than the time scale of molecular collisions, you can effectively treat the motion as random. The probability to move from a point $\mathbf{r}_{0}$ at time $t_{0}$ to a point $\mathbf{r}$ at a later time $t$ can then be expressed as a conditional probability distribution $P\left(\mathbf{r}, t \mid \mathbf{r}_{0}, t_{0}\right)$, which is referred to as the propagator. In a homogeneous medium, the propagator obeys the diffusion equation [14],

$$
\frac{\partial}{\partial t} P\left(\mathbf{r}, t \mid \mathbf{r}_{0}, t_{0}\right)=D \nabla_{\mathbf{r}}^{2} P\left(\mathbf{r}, t \mid \mathbf{r}_{0}, t_{0}\right),
$$

where $D$ is a constant known as the diffusivity. Given the initial position of a particle, $P\left(\mathbf{r}, t \mid \mathbf{r}, t_{0}\right)=\delta\left(\mathbf{r}-\mathbf{r}_{0}\right)$, the solution to equation (4.1) is

$$
P\left(\mathbf{r}, t \mid \mathbf{r}_{0}, t_{0}\right)=(4 \pi D t)^{-3 / 2} \exp \left(-\frac{\left(\mathbf{r}-\mathbf{r}_{0}\right)^{2}}{4 D t}\right) .
$$

This is also known as the fundamental solution, or Green's function, of the homogeneous diffusion equation.

At a fundamental level, the propagator depends on two opposing phenomena: the particle's limited kinetic energy, which restricts the distance it may travel (on average), and the increase in the possible number of trajectories that the particle can follow as the distance increases (effectively the entropy). This balance is exactly what the thermodynamic potential called the free energy describes. At equilibrium, the free energy is minimal [11]. By considering a small displacement of a particle in a system at equilibrium, Einstein was able to show $[13,17]$ that $D=k_{B} T / \zeta$, where $k_{B}$ is Boltzmann's constant, $T$ is the temperature and $\zeta$ is a property of the medium.

\subsubsection{Statistical ensembles}

In statistical physics, the concept of a statistical ensemble plays a key role. A statistical ensemble is the set of all replicas of the system, representing every possible state that the real system might be in. In other words, a statistical ensemble is a probability distribution for the state of the system. A system is said to be stationary if the probabilities of the ensemble states do not change with time. Given the ensemble of a system, it is possible to calculate the average value of any physical property $A$, as

$$
\langle A\rangle=\sum_{s} A(s) P(s)
$$

where $s$ is a possible state with probability $P(s)$. In this way, the ensemble average provides a bridge between the microscopic and the macroscopic properties of a system. 


\subsection{Stochastic process}

As we have seen above, the displacement of a particle from one instance in time to another can be considered a random variable. As time evolves, the particle will perform a sequence of random displacements. This is conveniently described using the concept of a stochastic process [69], which is the random function one obtains in limit of an infinite number of time steps. The mean of a stochastic process $x(t)$ is an average over the (possibly infinite) number of possible realizations, weighted by their corresponding probabilities

$$
\langle x(t)\rangle=\int x(t) P_{x}(x) d x .
$$

It can thus be interpreted as the average over an infinite number of realizations, i.e. the ensemble average. In general, the mean of a stochastic process is a function, however, for a stationary process it has a constant value. In that case one can subtract the mean value and it is therefore customary to assume that the mean is zero for a stationary process.

\subsubsection{Autocorrelation function}

By taking $n$ time samples, $t_{1}, \ldots, t_{n}$, we may define the $n$-th moment of the process as follows

$$
\left\langle x\left(t_{1}\right) \ldots x\left(t_{n}\right)\right\rangle=\int x\left(t_{1}\right) \ldots x\left(t_{n}\right) P_{x}(x) d x .
$$

Of particular interest is the autocorrelation function,

$$
\begin{aligned}
\Gamma_{x}\left(t_{1}, t_{2}\right) & =\left\langle\left(x\left(t_{1}\right)-\left\langle x\left(t_{1}\right)\right\rangle\right)\left(x\left(t_{2}\right)-\left\langle x\left(t_{2}\right)\right\rangle\right)\right\rangle \\
& =\left\langle x\left(t_{1}\right) x\left(t_{2}\right)\right\rangle-\left\langle x\left(t_{1}\right)\right\rangle\left\langle x\left(t_{2}\right)\right\rangle .
\end{aligned}
$$

For a stationary process, the autocorrelation function only depends on the time difference $\tau=\left|t_{1}-t_{2}\right|$ and hence, assuming zero mean, its autocorrelation function simplifies to

$$
\Gamma_{x}(\tau)=\langle x(\tau) x(0)\rangle .
$$

\subsubsection{Diffusivity and autocorrelation functions}

From the Gaussian nature of the propagator (4.2) it follows immediately that, in a homogeneous medium, $\left\langle\left(\mathbf{r}\left(t_{2}\right)-\mathbf{r}\left(t_{1}\right)\right)^{2}\right\rangle=6 D t$, and similarly in one dimension $\left\langle\left(x\left(t_{2}\right)-x\left(t_{1}\right)^{2}\right\rangle=2 D t\right.$. This can be used to define $D$ as follows,

$$
D=\lim _{t \rightarrow \infty} \frac{1}{2} \frac{d\left\langle X(t)^{2}\right\rangle}{d t},
$$


where we have introduced $X(t)=x(t)-x(0)$. Since $X(t)$ can be expressed in terms of the the velocity $v(t)$ as $X(t)=\int_{0}^{t} v\left(t^{\prime}\right) d t^{\prime}$, we have that

$$
\begin{aligned}
\frac{d\left\langle X(t)^{2}\right\rangle}{d t} & =\frac{d}{d t}\left\langle\left(\int_{0}^{t} v\left(t^{\prime}\right) d t^{\prime}\right)^{2}\right\rangle=2\left\langle v(t) \int_{0}^{t} v\left(t^{\prime}\right) d t^{\prime}\right\rangle \\
& =2 \int_{0}^{t}\left\langle v(t) v\left(t^{\prime}\right)\right\rangle d t^{\prime} .
\end{aligned}
$$

If we also assume that the process is stationary, then

$$
2 \int_{0}^{t}\left\langle v(t) v\left(t^{\prime}\right)\right\rangle d t^{\prime}=2 \int_{0}^{t}\langle v(\tau) v(0)\rangle d \tau=2 \int_{0}^{t} \Gamma_{v}(\tau) d \tau .
$$

So, under the assumption of stationarity, equation (4.9) can be written in terms of the velocity autocorrelation function as

$$
D=\lim _{t \rightarrow \infty} \int_{0}^{t} \Gamma_{v}(\tau) d \tau
$$

This result suggest that it may be useful to consider the time-dependent quantity

$$
D(t)=\int_{0}^{t} \Gamma_{v}(\tau) d \tau
$$

which has been termed the instantaneous diffusivity [51, 52]. The same authors also refer to the frequency domain counterpart of the velocity autocorrelation function,

$$
D(\omega)=\int_{0}^{\infty} \Gamma_{v}(\tau) e^{i \omega \tau} d \tau=\frac{1}{2} \int_{-\infty}^{\infty} \Gamma_{v}(\tau) e^{i \omega \tau} d \tau
$$

as the dispersive diffusivity. Here, the factor $1 / 2$ is due to causality: a reponse follows an excitation and cannot precede it, i.e. $\tau \geq 0$.

\subsubsection{The diffusion tensor}

The definition of the diffusivity (4.13) is straightforward to generalize to the case of anisotropic diffusion, i.e. when the diffusivity is different in different directions. This is done by overloading $D$ to also mean the diffusion tensor

$$
D=\left(\begin{array}{lll}
D_{x x} & D_{x y} & D_{x z} \\
D_{y x} & D_{y y} & D_{y z} \\
D_{z x} & D_{z y} & D_{z z}
\end{array}\right),
$$

with elements defined as

$$
\begin{aligned}
D_{\alpha \beta} & =\lim _{t \rightarrow \infty} \frac{1}{2} \frac{d\left\langle X_{\alpha}(t) X_{\beta}(t)\right\rangle}{d t} \\
& =\lim _{t \rightarrow \infty} \int_{0}^{t}\left\langle v_{\alpha}(\tau) v_{\beta}(0)\right\rangle d \tau .
\end{aligned}
$$


Clearly, $D$ is always symmetric. Whether $D$ refers to a scalar or a tensor should be clear from the context. The observation that diffusion is anisotropic in nerve tissue has been one of the main reasons for the interest in diffusion MRI [7, 8, 35, 41].

\subsection{Measuring diffusion with MRI}

In section 3.4 of the previous chapter, we saw that the addition of a spatially varying magnetic field $\mathbf{G}$ made the precession frequency vary spatially, according to

$$
\omega(\mathbf{r})=\gamma\left(B_{0}+\mathbf{r} \cdot \mathbf{G}\right) .
$$

Furthermore, we stated that, over time, a spin at position $\mathbf{r}$ would acquire a phase shift with respect to the rotating frame, given by

$$
\Phi(\mathbf{r}, t)=\int_{0}^{t} \gamma \mathbf{r} \cdot \mathbf{G}\left(t^{\prime}\right) d t^{\prime}=\mathbf{r} \cdot \gamma \int_{0}^{t} \mathbf{G}\left(t^{\prime}\right) d t .
$$

We will now revise that statement, because what has tacitly been assumed is that the spin is stationary and - given what we have learned about diffusion-we know better! To be clear about this distinction we will, in accordance with [13], henceforth use $\mathbf{g}(t)$, instead of $\mathbf{G}(\mathrm{t})$, to denote a time-varying gradient that is used to encode for motion. Moreover, we will include any spin reversals due to RF pulses through a sign change in $\mathbf{g}(t)$, so that it represents the effective gradient experienced by the spins. The diffusion measurements we will consider typically involve some type of echo (cf. section 3.3.2), which means that at the echo time, $T_{E}$, we have that $\int_{0}^{T_{E}} g\left(t^{\prime}\right) d t^{\prime}=0$.

To measure the total magnetization, it is convenient to use the concept of a spin packet, which is defined as all spins excited at a given point $\mathbf{r}_{0}$ at the initial time moment $t=0$. At a later time $t$, the contribution to the signal from a spin packet is given by

$$
S\left(\mathbf{r}_{0}, t\right)=\rho\left(\mathbf{r}_{0}\right)\left\langle\exp \left(i \gamma \int_{0}^{t} \mathbf{g}\left(t^{\prime}\right) \cdot \mathbf{r}\left(t^{\prime}\right) d t^{\prime}\right)\right\rangle \exp \left(-t / T_{2}\right),
$$

where the ensemble average is a sum over the infinite number of possible trajectories of a diffusing particle starting at $\mathbf{r}_{0}$ and diffusing for a time $t$. This equation is fundamental to diffusion MRI and we will have reason to return to it on multiple occasions.

The total signal is found by integrating over all starting positions,

$$
S(t)=\int S\left(\mathbf{r}_{0}, t\right) d \mathbf{r}_{0} .
$$




\subsubsection{Eulerian and Lagrangian description of flow}

There are two complementary ways of describing flow [40]. In the Lagrangian description, particles are followed as they move through a flow field. As a particle moves, its velocity will fluctuate, and can thus be considered a stochastic process. In the Eulerian description, a flow's characteristics is monitored at fixed locations and can therefore be expected to vary smoothly in most cases. In light of these definitions, it is clear that the ensemble average (4.21) is formulated using a Lagrangian perspective.

\subsubsection{The Bloch-Torrey equation}

Suppose instead that we consider the transverse magnetization $m(\mathbf{r}, t)$ at a fixed point in space, i.e. using an Eulerian description. The ensemble average then amounts to an integral over the spatial dimensions. From this perspective, it can be shown [68], that the time-evolution of the magnetization can be described by incorporating a diffusion term into the Bloch equation so that, in the rotating frame,

$$
\frac{\partial m}{\partial t}=\nabla \cdot D(\mathbf{r}) \nabla m-i \gamma \mathbf{g} \cdot \mathbf{r}-\frac{m}{T_{2}} .
$$

This is referred to as the Bloch-Torrey equation. In a theoretical analysis, the transverse relaxation is often neglected. To see why, we make the ansatz $m(\mathbf{r}, t)=\tilde{m}(\mathbf{r}, t) e^{-t / T_{2}}$, and insert in the Bloch-Torrey equation. This yields,

$$
\begin{aligned}
& \frac{\partial \tilde{m}}{\partial t}-\frac{\tilde{m}}{T_{2}}=\nabla \cdot D(\mathbf{r}) \nabla \tilde{m}-i \gamma \mathbf{g} \cdot \mathbf{r}-\frac{\tilde{m}}{T_{2}} \\
& \Longleftrightarrow \frac{\partial \tilde{m}}{\partial t}=\nabla \cdot D(\mathbf{r}) \nabla \tilde{m}-i \gamma \mathbf{g} \cdot \mathbf{r} .
\end{aligned}
$$

So, when we are only interested in the effects of the applied gradient it is common to normalize the signal to the case $\mathbf{g}(t)=0$. This is defined as the normalized echo amplitude,

$$
E(t)=\frac{S(t)_{\mathbf{g}(t) \neq 0}}{\left.S_{(}\right)_{\mathbf{g}(t)=0}} .
$$

In a few cases, the Bloch-Torrey equation can be solved analytically. One such case is for a constant scalar diffusivity $D$. Then, the ansatz

$$
m(\mathbf{r}, t)=A(t) \exp \left(-i \gamma \mathbf{r} \cdot \int_{0}^{t} \mathbf{g}\left(t^{\prime}\right) d t^{\prime}\right) \exp \left(-t / T_{2}\right)
$$

reduces the Bloch-Torrey equation to an ordinary differential equation for $A(t)$. This, in turn, is solved by

$$
A(t)=\exp \left(-D \int_{0}^{t} \mathbf{q}\left(t^{\prime}\right)^{T} \mathbf{q}\left(t^{\prime}\right) d t^{\prime}\right)
$$


where we have introduced

$$
\mathbf{q}(t)=\gamma \int_{0}^{t} \mathbf{g}(t)
$$

Note that at the echo center, the complex exponential in equation (4.27) disappears, and the normalized echo amplitude is thus

$$
E\left(T_{E}\right)=\exp \left(-D \int_{0}^{T_{E}} \mathbf{q}\left(t^{\prime}\right)^{2} d t^{\prime}\right) .
$$

Following an identical line of reasoning, one may show [13] that in the case of a constant diffusion tensor

$$
E\left(T_{E}\right)=\exp \left(-\int_{0}^{T_{E}} \mathbf{q}\left(t^{\prime}\right)^{T} D \mathbf{q}\left(t^{\prime}\right) d t^{\prime}\right) .
$$

Paper III describes a method of numerically optimizing the gradient waveform $\mathbf{g}(t)$ (or, equivalently, $\mathbf{q}(t)$ ) to achieve maximum diffusion weighting subject to constraints imposed by the MR scanner.

\subsection{The Stejskal-Tanner experiment}

In 1965, Stejskal and Tanner demonstrated [62] what remains to this day the predominant diffusion measurement using magnetic resonance: the pulsed field gradient (PFG). Figure 4.1 shows an example of a PFG sequence that uses a spin echo. Although details in the implementation may vary, the main characteristic of a PFG measurement it that the effective gradient sequence consists of two blocks of equal magnitudes but opposite directions. The gradient sequence can thus be written

$$
\mathbf{g}(t)= \begin{cases}-\mathbf{g} & t_{1} \leq t \leq t_{1}+\delta \\ \mathbf{g} & t_{1}+\Delta \leq t \leq t_{1}+\Delta+\delta \\ 0 & \text { otherwise }\end{cases}
$$

Inserting this into (4.31), we find that the signal at the echo time is given by

$$
E_{\mathrm{PFG}}\left(T_{E}\right)=\exp \left(-\gamma^{2} \delta^{2}(\Delta-\delta / 3) \mathbf{g}^{T} D \mathbf{g}\right)
$$

For this reason, the echo attenuation (diffusion weighting) is often expressed using the $b$-value, defined as

$$
b=\gamma^{2}\|\mathbf{g}\|^{2} \delta^{2}(\Delta-\delta / 3) .
$$

Obviously, this definition presupposes that a PFG sequence is used.

By repeating the PFG experiment with different gradient directions, it is possible to estimate the full diffusion tensor. This is referred to as diffusion tensor imaging (DTI) [7]. 


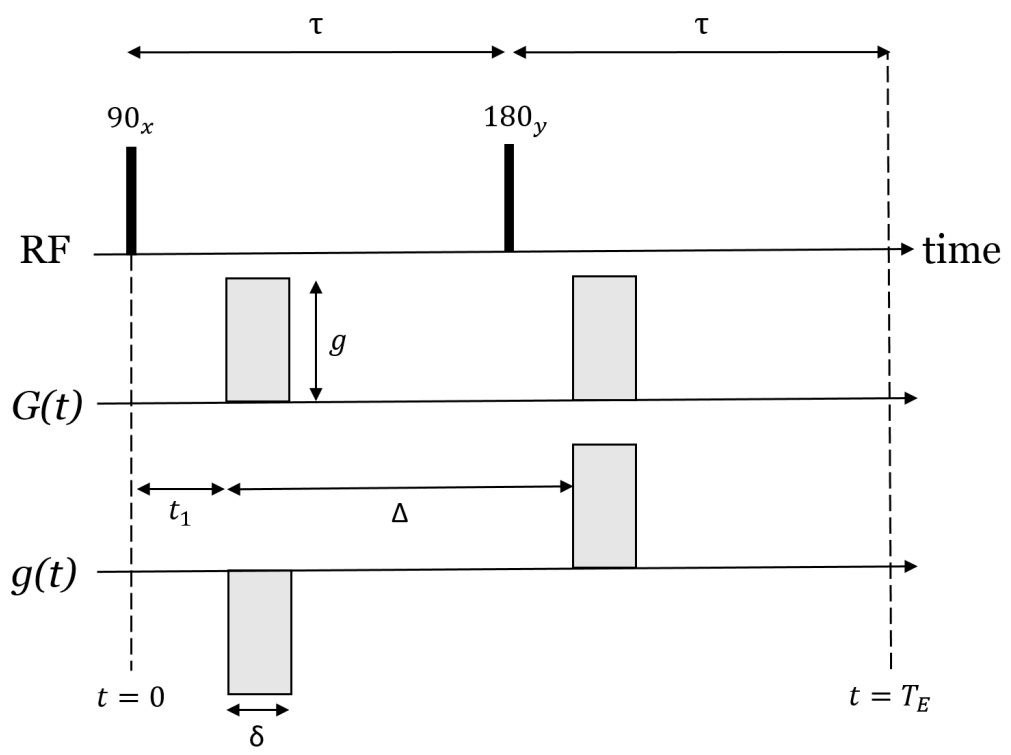

Figure 4.1: The single pulsed field gradient experiment-the mainstay of diffusion MRI. The top line shows the RF excitations, the middle line the actual gradient sequence applied and the bottom line the effective gradient sequence (with sign change at $180^{\circ}$ pulse). 


\subsubsection{The narrow pulse approximation}

When the diffusivity is not constant it is in most cases impossible to obtain a closed form solution to the Bloch-Torrey equation. An important exception is for a PFG experiment where the gradient duration $\delta$ is so short that motion during its application can be neglected. This is referred to as the narrow pulse approximation. Mathematically, it implies that the gradient sequence can be approximated as the sum of two Dirac pulses

$$
\mathbf{g}(t)=\mathbf{q}\left[\delta\left(t-\left(t_{1}+\Delta\right)\right)-\delta\left(t-t_{1}\right)\right],
$$

where, in this expression, $\mathbf{q}=\gamma \delta \mathbf{g}$ is a constant. This notation may be confusing, but the reason we use it anyway is that imaging under the narrow pulse approximation has an established name: q-space imaging. Referring back to equation (4.21), the contribution to the signal from a spin-packet subject to this gradient sequence is

$$
S\left(\mathbf{r}_{0}, T_{E}\right)=\rho\left(\mathbf{r}_{0}\right)\left\langle e^{i \mathbf{q} \cdot\left(\mathbf{r}\left(t_{1}+\Delta\right)-\mathbf{r}\left(t_{1}\right)\right)}\right\rangle e^{-T_{E} / T_{2}} .
$$

So, the ensemble average over the whole stochastic process $\mathbf{r}(t)$ is effectively reduced to an average over the difference at the two sample times $t=t_{1}$ and $t=t_{1}+\Delta$. To simplify, we let $\mathbf{r}_{1}=\mathbf{r}\left(t_{1}\right), \mathbf{r}_{2}=\mathbf{r}\left(t_{1}+\Delta\right)$ and $\mathbf{R}=\mathbf{r}\left(t_{1}+\Delta\right)-\mathbf{r}\left(t_{1}\right)$. Further we define the average propagator,

$$
\bar{P}(\mathbf{R}, \Delta)=\int P\left(\mathbf{r}_{1}+\mathbf{R} \mid \mathbf{r}_{1}\right) P\left(\mathbf{r}_{1}\right) d \mathbf{r}_{1} .
$$

This allows us to write

$$
\begin{aligned}
E(\mathbf{q}, \Delta) & =\frac{\int S\left(\mathbf{r}_{0}, T_{E}\right) d \mathbf{r}_{0}}{\int \rho\left(\mathbf{r}_{0}\right) e^{-T_{E} / T_{2}} d \mathbf{r}_{0}}=\left\langle e^{i \mathbf{q} \cdot\left(\mathbf{r}_{2}-\mathbf{r}_{1}\right)}\right\rangle \\
& =\iint P\left(\mathbf{r}_{1}, \mathbf{r}_{2}\right) e^{i \mathbf{q} \cdot\left(\mathbf{r}_{2}-\mathbf{r}_{1}\right)} d \mathbf{r}_{1} d \mathbf{r}_{2} \\
& =\iint P\left(\mathbf{r}_{2} \mid \mathbf{r}_{1}\right) P\left(\mathbf{r}_{1}\right) e^{i \mathbf{q} \cdot\left(\mathbf{r}_{2}-\mathbf{r}_{1}\right)} d \mathbf{r}_{1} d \mathbf{r}_{2} \\
& =\int\left(\int P\left(\mathbf{r}_{1}\right) P\left(\mathbf{r}_{1}+\mathbf{R} \mid \mathbf{r}_{1}\right) d \mathbf{r}_{1}\right) e^{i \mathbf{q} \cdot \mathbf{R}} d \mathbf{R} \\
& =\int \bar{P}(\mathbf{R}) e^{i \mathbf{q} \cdot \mathbf{R}} d \mathbf{R} .
\end{aligned}
$$

In other words, the echo amplitude in a q-space imaging experiment is nothing but the Fourier transform of the average propagator (4.37). This holds true without any assumptions on the underlying diffusivity. In the case of a constant diffusion tensor, the echo amplitude can also be expressed as in (4.33), which in the narrow pulse approximation is modified to read

$$
E\left(T_{E}\right)=\exp \left(-\gamma^{2} \delta^{2} \Delta \mathbf{g}^{T} D \mathbf{g}\right)=\exp \left(-\Delta \mathbf{q}^{T} D \mathbf{q}\right) .
$$


This allows us to determine the average propagator by inverse Fourier transforming equation (4.42), which gives

$$
\bar{P}(\mathbf{R}, \Delta)=\frac{1}{(4 \pi \Delta)^{3 / 2}|D|^{1 / 2}} \exp \left(\frac{1}{4 \Delta} \mathbf{R}^{T} D^{-1} \mathbf{R}\right) .
$$

In other words, it is the same as the propagator for free diffusion (4.2) (modified to an anisotropic medium). This is perhaps not surprising-the assumption that the diffusion tensor is constant implies, after all, that the propagator is everywhere the same.

In the limit $\|\mathbf{q}\| \rightarrow 0$, a Taylor expansion of equation (4.42) gives

$$
E(\mathbf{q}, \Delta) \approx \int \bar{P}(\mathbf{R})\left(1+i \mathbf{q} \cdot \mathbf{R}-\frac{1}{2}(\mathbf{q} \cdot \mathbf{R})^{2}\right) d \mathbf{R} .
$$

If there is no net flow the linear term disappears, and we have that

$$
E(\mathbf{q}, \Delta) \approx 1-\frac{\|q\|^{2}}{2} \int \bar{P}(\mathbf{R})(\mathbf{q} \cdot \mathbf{R})^{2} d \mathbf{R}=1-\frac{1}{2}\left\langle(\mathbf{q} \cdot \mathbf{R})^{2}\right\rangle .
$$

This means that - given that the narrow pulse approximation is valid-the low $q$-regime can be used to estimate the mean-square displacement.

\subsubsection{The apparent diffusion coefficient}

Most of the results derived up to this point have assumed that the diffusing particles are free to move without surfaces or boundaries restricting them. In such cases we expect that the average propagator is a Gaussian, as shown in the previous section. When diffusion is restricted, this is no longer true. Of course it is still possible to fit the echo amplitude with an expression of the form $e^{-b D_{\mathrm{app}}}$, and many people do, but the precise meaning of the apparent diffusion coefficient $D_{\text {app }}$ (also known as ADC) is in most cases unclear [24]. The apparent diffusion coefficient is not an intrinsic property of the medium, but depends on the details of the experiment. Thus, reporting the value of $D_{\text {app }}$ without an accurate description of the experimental procedure is, strictly speaking, nonsensical. Nevertheless, use-and often misuse - of the apparent diffusion coefficient is widespread in the clinical MRI literature [24, 71]. 


\section{Bibliography}

[1] A. Abragam. The Principles of Nuclear Magnetism. International series of monographs on physics. Clarendon Press, 1961.

[2] A. Ahnesjö and M. M. Aspradakis. Dose calculations for external photon beams in radiotherapy. Physics in Medicine and Biology, 44(11):R99-R155, 1999.

[3] A. Ahnesjö. Collapsed cone convolution of radiant energy for photon dose calculation in heterogeneous media. Medical physics, 16(4):577$592,1989$.

[4] A. Ahnesjö, M. Saxner, and A. Trepp. A pencil beam model for photon dose calculation. Medical physics, 19(2):263-273, 1992.

[5] B. Alberts, D. Bray, K. Hopkin, A. Johnson, J. Lewis, M. Raff, K. Roberts, and P. Walter. Essential cell biology. Garland Science, 3rd edition, 2013.

[6] D. W. Andrews, C. B. Scott, P. W. Sperduto, A. E. Flanders, L. E. Gaspar, M. C. Schell, M. Werner-Wasik, W. Demas, J. Ryu, J.-P. Bahary, et al. Whole brain radiation therapy with or without stereotactic radiosurgery boost for patients with one to three brain metastases: phase III results of the RTOG 9508 randomised trial. The Lancet, 363(9422):1665-1672, 2004.

[7] P. J. Basser, J. Mattiello, and D. LeBihan. MR diffusion tensor spectroscopy and imaging. Biophysical journal, 66(1):259, 1994.

[8] C. Beaulieu. The basis of anisotropic water diffusion in the nervous system-a technical review. NMR in Biomedicine, 15(7-8):435-455, 2002 .

[9] S. H. Benedict, K. M. Yenice, D. Followill, J. M. Galvin, W. Hinson, B. Kavanagh, P. Keall, M. Lovelock, S. Meeks, L. Papiez, et al. Stereotactic body radiation therapy: the report of AAPM Task Group 101. Medical physics, 37(8):4078-4101, 2010.

[10] J. Bernier, E. J. Hall, and A. Giaccia. Radiation oncology: a century of achievements. Nature Reviews Cancer, 4(9):737-747, 2004. 
[11] R. Bowley and M. Sanchez. Introductory statistical mechanics. Clarendon Press Oxford, 1999.

[12] J. M. Brown, D. J. Carlson, and D. J. Brenner. The tumor radiobiology of SRS and SBRT: are more than the 5 R's involved? International Journal of Radiation Oncology* Biology* Physics, 88(2):254-262, 2014.

[13] P. T. Callaghan. Translational dynamics and magnetic resonance: principles of pulsed gradient spin echo NMR. Oxford University Press, 2011.

[14] S. Chandrasekhar. Stochastic problems in physics and astronomy. Reviews of modern physics, 15(1):1, 1943.

[15] L. Chen, R. Price Jr, T. Nguyen, L. Wang, J. Li, L. Qin, M. Ding, E. Palacio, C. Ma, and A. Pollack. Dosimetric evaluation of MRIbased treatment planning for prostate cancer. Physics in medicine and biology, 49(22):5157, 2004.

[16] J. Deacon, M. Peckham, and G. Steel. The radioresponsiveness of human tumours and the initial slope ofthe cell survival curve. Radiotherapy and Oncology, 2(4):317-323, 1984.

[17] A. Einstein. Über die von der molekularkinetischen theorie der wärme geforderte bewegung von in ruhenden flüssigkeiten suspendierten teilchen. Annalen der physik, 322(8):549-560, 1905.

[18] A. Eklund. Signal processing for robust and real-time fMRI with application to brain computer interfaces, 2010. Licentiate thesis.

[19] B. Fertil and E.-P. Malaise. Inherent cellular radiosensitivity as a basic concept for human tumor radiotherapy. International Journal of Radiation Oncology* Biology* Physics, 7(5):621-629, 1981.

[20] M. Fippel. Fast Monte Carlo dose calculation for photon beams based on the VMC electron algorithm. Medical Physics, 26(8):1466-1475, 1999.

[21] D. Formica and S. Silvestri. Biological effects of exposure to magnetic resonance imaging: an overview. Biomedical engineering online, $3(1): 11,2004$.

[22] M. Garcia-Barros, F. Paris, C. Cordon-Cardo, D. Lyden, S. Rafii, A. Haimovitz-Friedman, Z. Fuks, and R. Kolesnick. Tumor response to radiotherapy regulated by endothelial cell apoptosis. Science, 300(5622):1155-1159, 2003.

[23] L. H. Gray, A. Conger, M. Ebert, S. Hornsey, and O. Scott. The concentration of oxygen dissolved in tissues at the time of irradiation as 
a factor in radiotherapy. The British journal of radiology, 26(312):638648, 1953.

[24] D. S. Grebenkov. Use, misuse, and abuse of apparent diffusion coefficients. Concepts in Magnetic Resonance Part A, 36(1):24-35, 2010.

[25] D. J. Griffiths. Introduction to quantum mechanics. Pearson Education International, 2nd edition, 2005.

[26] E. M. Haacke, R. W. Brown, M. R. Thompson, and R. Venkatesan. Magnetic resonance imaging: physical principles and sequence design. Wiley-Liss, 1999.

[27] E. L. Hahn. Spin echoes. Physical Review, 80(4):580, 1950.

[28] E. J. Hall and A. J. Giaccia. Radiobiology for the Radiologist. Lippincott Williams \& Wilkins, 2006.

[29] D. Hanahan and R. A. Weinberg. The hallmarks of cancer. Cell, 100(1):57-70, 2000.

[30] D. Hanahan and R. A. Weinberg. Hallmarks of cancer: the next generation. cell, 144(5):646-674, 2011.

[31] S. Hissoiny, B. Ozell, H. Bouchard, and P. Després. GPUMCD: A new GPU-oriented Monte Carlo dose calculation platform. Medical physics, $38(2): 754-764,2011$.

[32] M. Höckel and P. Vaupel. Tumor hypoxia: definitions and current clinical, biologic, and molecular aspects. Journal of the National Cancer Institute, 93(4):266-276, 2001.

[33] International Commission on Radiation Units and Measurements. Tissue Substitutes in Radiation Dosimetry and Measurement, 1989. Report 44 .

[34] International Commission on Radiation Units and Measurements. Photon, Electron, Proton and Neutron Interaction Data for Body Tissues, 1992. Report 46.

[35] B. J. Jellison, A. S. Field, J. Medow, M. Lazar, M. S. Salamat, and A. L. Alexander. Diffusion tensor imaging of cerebral white matter: a pictorial review of physics, fiber tract anatomy, and tumor imaging patterns. American Journal of Neuroradiology, 25(3):356-369, 2004.

[36] H. Jiang, J. Seco, and H. Paganetti. Effects of Hounsfield number conversion on CT based proton Monte Carlo dose calculations. Medical physics, 34(4):1439-1449, 2007. 
[37] I. Kawrakow, M. Fippel, and K. Friedrich. 3D electron dose calculation using a Voxel based Monte Carlo algorithm (VMC). Medical Physics, 23(4):445-457, 1996.

[38] D. Kondziolka, S. M. Shin, A. Brunswick, I. Kim, and J. S. Silverman. The biology of radiosurgery and its clinical applications for brain tumors. Neuro-oncology, 17(1):29-44, 2015.

[39] T. Krieger and O. A. Sauer. Monte Carlo-versus pencil-beam/collapsed-cone-dose calculation in a heterogeneous multi-layer phantom. Physics in medicine and biology, 50(5):859, 2005.

[40] P. K. Kundu, I. M. Cohen, and D. R. Dowling. Fluid mechanics. Elsevier, 5th edition, 2012.

[41] D. Le Bihan. Looking into the functional architecture of the brain with diffusion MRI. Nature Reviews Neuroscience, 4(6):469-480, 2003.

[42] L. Leksell. The stereotaxic method and radiosurgery of the brain. Acta Chir. Scand., 102:316-319, 1951.

[43] Z.-P. Liang and P. C. Lauterbur. Principles of magnetic resonance imaging. SPIE Optical Engineering Press, 2000.

[44] M. E. Linskey, D. W. Andrews, A. L. Asher, S. H. Burri, D. Kondziolka, P. D. Robinson, M. Ammirati, C. S. Cobbs, L. E. Gaspar, J. S. Loeffler, et al. The role of stereotactic radiosurgery in the management of patients with newly diagnosed brain metastases: a systematic review and evidence-based clinical practice guideline. Journal of neuro-oncology, 96(1):45-68, 2010.

[45] L. D. Lunsford, D. Kondziolka, J. C. Flickinger, D. J. Bissonette, C. A. Jungreis, A. H. Maitz, J. A. Horton, and R. J. Coffey. Stereotactic radiosurgery for arteriovenous malformations of the brain. Journal of neurosurgery, 75(4):512-524, 1991.

[46] S. Maesawa, C. Salame, J. C. Flickinger, S. Pirris, D. Kondziolka, and L. D. Lunsford. Clinical outcomes after stereotactic radiosurgery for idiopathic trigeminal neuralgia. Journal of neurosurgery, 94(1):14-20, 2001.

[47] N. Matsufuji, H. Tomura, Y. Futami, H. Yamashita, A. Higashi, S. Minohara, M. Endo, and T. Kanai. Relationship between CT number and electron density, scatter angle and nuclear reaction for hadron-therapy treatment planning. Physics in medicine and biology, 43(11):3261, 1998.

[48] S. Mattsson, A. Brahme, J. Carlsson, J. Denekamp, E. ForssellAronsson, M. Hellström, K.-A. Johansson, E. Kjellén, B. Littbrand, 
B. Nordenskjöld, et al. Swedish Cancer Society radiation therapy research investigation. Acta Oncologica, 41(7):596-603, 2002.

[49] D. W. McRobbie, E. A. Moore, M. J. Graves, and M. R. Prince. MRI from Picture to Proton. Cambridge university press, 2006.

[50] A. Norlund. Costs of radiotherapy. Acta Oncologica, 42(5-6):411-415, 2003.

[51] D. S. Novikov, J. H. Jensen, J. A. Helpern, and E. Fieremans. Revealing mesoscopic structural universality with diffusion. Proceedings of the National Academy of Sciences, 111(14):5088-5093, 2014.

[52] D. S. Novikov and V. G. Kiselev. Effective medium theory of a diffusion-weighted signal. NMR in biomedicine, 23(7):682-697, 2010.

[53] H. J. Park, R. J. Griffin, S. Hui, S. H. Levitt, and C. W. Song. Radiation-induced vascular damage in tumors: implications of vascular damage in ablative hypofractionated radiotherapy (SBRT and SRS). Radiation research, 177(3):311-327, 2012.

[54] R. Prabhakar, P. Julka, T. Ganesh, A. Munshi, R. Joshi, and G. Rath. Feasibility of using mri alone for $3 \mathrm{~d}$ radiation treatment planning in brain tumors. Japanese journal of clinical oncology, 37(6):405-411, 2007 .

[55] K. M. Prise, G. Schettino, M. Folkard, and K. D. Held. New insights on cell death from radiation exposure. The lancet oncology, 6(7):520-528, 2005 .

[56] U. Ringborg, D. Bergqvist, B. Brorsson, E. Cavallin-Ståhl, J. Ceberg, N. Einhorn, J.-e. Frödin, J. Järhult, G. Lamnevik, C. Lindholm, et al. The Swedish Council on Technology Assessment in Health Care (SBU) systematic overview of radiotherapy for cancer including a prospective survey of radiotherapy practice in Sweden 2001-summary and conclusions. Acta oncologica, 42(5-6):357-365, 2003.

[57] M. D. Robson, P. D. Gatehouse, M. Bydder, and G. M. Bydder. Magnetic resonance: an introduction to ultrashort TE (UTE) imaging. Journal of computer assisted tomography, 27(6):825-846, 2003.

[58] W. Schneider, T. Bortfeld, and W. Schlegel. Correlation between CT numbers and tissue parameters needed for Monte Carlo simulations of clinical dose distributions. Physics in medicine and biology, 45(2):459, 2000 .

[59] J. Seco and P. Evans. Assessing the effect of electron density in photon dose calculations. Medical physics, 33(2):540-552, 2006. 
[60] J. Seco and F. Verhaegen. Monte Carlo techniques in radiation therapy. CRC Press, 2013.

[61] G. G. Steel, T. J. McMillan, and J. Peacock. The 5Rs of radiobiology. International journal of radiation biology, 56(6):1045-1048, 1989.

[62] E. Stejskal and J. Tanner. Spin diffusion measurements: spin echoes in the presence of a time-dependent field gradient. The journal of chemical physics, 42(1):288-292, 1965.

[63] H. Svensson and T. R. Möller. Developments in radiotherapy. Acta Oncologica, 42(5-6):430-442, 2003.

[64] Swedish Radiation Safety Authority. Report from SSM's scientific council on ionizing radiation within oncology, 2010, 2011. Report 2011:25.

[65] Swedish Radiation Safety Authority. Report from SSM's scientific council on ionizing radiation within oncology, 2013. MR in radiotherapy - an important step towards personalised treatment?, 2014. Report 2014:51.

[66] M. Talbäck and P. W. Dickman. Predicting the survival of cancer patients recently diagnosed in Sweden and an evaluation of predictions published in 2004. Acta Oncologica, 51(1):17-27, 2012.

[67] M. Talbäck, M. Rosén, M. Stenbeck, and P. W. Dickman. Cancer patient survival in Sweden at the beginning of the third millenniumpredictions using period analysis. Cancer Causes $\&$ Control, 15(9):967976, 2004.

[68] H. C. Torrey. Bloch equations with diffusion terms. Physical Review, 104(3):563, 1956.

[69] N. G. Van Kampen. Stochastic processes in physics and chemistry. North Holland, 3rd edition, 2007.

[70] P. Vaupel. Tumor microenvironmental physiology and its implications for radiation oncology. In Seminars in radiation oncology, volume 14, pages 198-206. Elsevier, 2004.

[71] M. Vermoolen, T. Kwee, and R. Nievelstein. Apparent diffusion coefficient measurements in the differentiation between benign and malignant lesions: a systematic review. Insights into imaging, 3(4):395-409, 2012 .

[72] J. F. Ward. Nature of lesions formed by ionizing radiation. In DNA damage and repair, pages 65-84. Springer, 1998. 
[73] M. Weiger, D. O. Brunner, B. E. Dietrich, C. F. Müller, and K. P. Pruessmann. ZTE imaging in humans. Magnetic Resonance in Medicine, 70(2):328-332, 2013.

[74] F. Wiesinger, L. I. Sacolick, A. Menini, S. S. Kaushik, S. Ahn, P. VeitHaibach, G. Delso, and D. D. Shanbhag. Zero TE MR bone imaging in the head. Magnetic Resonance in Medicine, 2015. In press.

[75] H. R. Withers. The four R's of radiotherapy. Adv Radiat Biol, 5(3):241271, 1975.

[76] Y. Xiao, L. Papiez, R. Paulus, R. Timmerman, W. L. Straube, W. R. Bosch, J. Michalski, and J. M. Galvin. Dosimetric evaluation of heterogeneity corrections for RTOG 0236: Stereotactic body radiotherapy of inoperable stage I-II non-small-cell lung cancer. International Journal of Radiation Oncology* Biology* Physics, 73(4):1235-1242, 2009. 



\section{Papers}

The articles associated with this thesis have been removed for copyright reasons. For more details about these see:

http://urn.kb.se/resolve?urn=urn:nbn:se:liu:diva-115796 